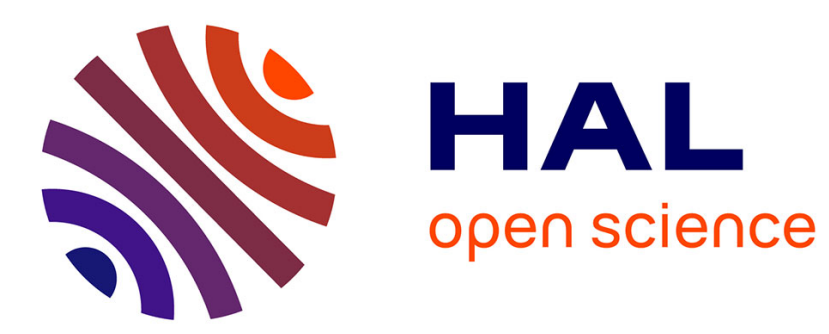

\title{
High-Order Gradients with the Shifted Boundary Method: An Embedded Enriched Mixed Formulation for Elliptic PDEs
}

Leo Nouveau, Mario Ricchiuto, Guglielmo Scovazzi

\section{- To cite this version:}

Leo Nouveau, Mario Ricchiuto, Guglielmo Scovazzi. High-Order Gradients with the Shifted Boundary Method: An Embedded Enriched Mixed Formulation for Elliptic PDEs. Journal of Computational Physics, 2019, pp.108898. 10.1016/j.jcp.2019.108898 . hal-02269007

\section{HAL Id: hal-02269007 https://hal.inria.fr/hal-02269007}

Submitted on 22 Aug 2019

HAL is a multi-disciplinary open access archive for the deposit and dissemination of scientific research documents, whether they are published or not. The documents may come from teaching and research institutions in France or abroad, or from public or private research centers.
L'archive ouverte pluridisciplinaire HAL, est destinée au dépôt et à la diffusion de documents scientifiques de niveau recherche, publiés ou non, émanant des établissements d'enseignement et de recherche français ou étrangers, des laboratoires publics ou privés. 


\title{
High-Order Gradients with the Shifted Boundary Method: An Embedded Enriched Mixed Formulation for Elliptic PDEs
}

\author{
L. Nouveau ${ }^{\mathrm{a}}$, M. Ricchiuto ${ }^{\mathrm{b}}$, G. Scovazzi ${ }^{\mathrm{a}, *}$ \\ ${ }^{a}$ Department of Civil and Environmental Engineering, Duke University, Durham, North Carolina 27708 - USA \\ ${ }^{b}$ Team CARDAMOM, INRIA Bordeaux Sud Ouest, 200 Avenue de la Vieille Tour, 33405 Talence cedex - France
}

\begin{abstract}
We propose an extension of the embedded boundary method known as "shifted boundary method" to elliptic diffusion equations in mixed form (e.g., Darcy flow, heat diffusion problems with rough coefficients, etc.). Our aim is to obtain an improved formulation that, for linear finite elements, is at least second-order accurate for both flux and primary variable, when either Dirichlet or Neumann boundary conditions are applied. Following previous work of Nishikawa and Mazaheri in the context of residual distribution methods, we consider the mixed form of the diffusion equation (i.e., with Darcy-type operators), and introduce an enrichment of the primary variable. This enrichment is obtained exploiting the relation between the primary variable and the flux variable, which is explicitly available at nodes in the mixed formulation. The proposed enrichment mimics a formally quadratic pressure approximation, although only nodal unknowns are stored, similar to a linear finite element approximation. We consider both continuous and discontinuous finite element approximations and present two approaches: a non-symmetric enrichment, which, as in the original references, only improves the consistency of the overall method; and a symmetric enrichment, which enables a full error analysis in the classical finite element context. Combined with the shifted boundary method, these two approaches are extended to high-order embedded computations, and enable the approximation of both primary and flux (gradient) variables with second-order accuracy, independently on the type of boundary conditions applied. We also show that the the primary variable is third-order accurate, when pure Dirichlet boundary conditions are embedded.
\end{abstract}

Keywords: Darcy flow; embedded boundary; finite element method; high-order approximation; stabilized methods; computational fluid dynamics.

\section{Introduction}

The proposed work stems from the Shifted Boundary Method (SBM), an embedded method that has been recently developed and applied in the context of the Laplace and Stokes problems [28], the Navier-Stokes equations [29] and hyperbolic systems [46]. The SBM leverages a surrogate/approximate interface representation, by which the location where boundary conditions are applied is shifted from the true to the surrogate interface, and, most importantly, a Taylor expansion is used to modify the value of boundary conditions, with the goal of preventing a reduction in the convergence rates of the overall formulation. The appropriate (modified) boundary conditions are applied weakly, using, for example, a Nitsche strategy (other weak enforcement strategies are, in principle, also possible).

Immersed and embedded boundary methods present several advantages with respect to conformal methods especially regarding mesh generation. If complex geometries are involved, generating the mesh can require considerable time and effort compared to the whole simulation process. Introduced by Peskin in 1972 [40], the Immersed Boundary Method (IBM) proposes to mesh the entire computational domain, independently of the geometry of the problem. Bodies or boundaries present in the domain are described implicitly in the mesh by means of a distance function, or

\footnotetext{
*Corresponding author: Guglielmo Scovazzi. Email: guglielmo.scovazzi@ duke.edu, Phone: +1 (919) 660-5075, Fax: +1 (919) 660-5219

Email addresses: leo.nouveau@duke.edu (L. Nouveau), mario.ricchiuto@inria.fr (M. Ricchiuto), guglielmo.scovazzi@duke.edu (G. Scovazzi )
} 
similar indicator function. The main idea behind the IBM is to solve the model equations on the entire domain, and to impose the boundary conditionss via a forcing term. The method was originally designed on Cartesian grids and its development has been an active field of research for some decades now. Two exhaustive reviews were written by Mittal and Iaccarino in 2005 [34], and Sotiropoulos and Yang in 2014 [47]. The main drawback of the IBM is the accuracy of boundary conditions, which are most often only first-order accurate. Some strategies have been proposed in the past to increase the IBM accuracy, at the expense of more involved implementations [18, 17, 26, 27], or to compensate low-order accuracy by means of mesh adaptation [38, 6, 19].

A similar objective is pursued by Embedded Boundary Methods, which still use an immersed description of bodies and boundaries, but solve the model equations only in the regions of interest. Within a finite element context, the cut-cell and cutFEM approach [39, 15, 20, 10, 24, 48] are most commonly utilized. These techniques usually combine a weak enforcement of the boundary conditions with a XFEM strategy. For example, in CutFEMs, the boundary is reconstructed as the intersection between the boundary surface and the embedding (underlaying) grid. Approaches of this kind often require complex implementations and may suffer from the so-called small-cut cell issue, unless specific stabilization mechanisms are put in place [7, 8, 44, 43]. In fact, the intersection of the grid with the surface boundary may produce elements of extremely small sizes, with negative consequences on the stability of the variational formulation and the magnitude of the condition number of the associated algebraic system.

The SBM is effective in overcoming all these issues, and proved robust and accurate in the case of Dirichlet conditions, while in the case of Neumann conditions, a standard SBM implementation may not be optimally accurate, since the Taylor expansion of the gradient of the solution is required, and this is not feasible for the simple linear finite element approximation. In [28], for the Laplace problem, the use of a reconstructed gradient has been used to overcome this difficulty.

A more robust strategy to achieve accurate gradients is pursued here, and consists in developing a mixed formulation, in which the Poisson operator is replaced by the Darcy operator, and to design advanced schemes for high-order flux approximations. In this context, we would like to mention recent contributions [35, 31, 32, 33] for body-fitted computations, which were inspired by the earlier work of Caraeni [9]. In these works, mixed forms of the Poisson, diffusion, or advection diffusion operators were proposed, in which the solution derivatives are adopted as main unknowns, together with the solution itself. This procedure enhances the accuracy of the solution, and allows to obtain uniformly second-order accurate derivatives of the solution. An additional pressure enrichment was also proposed in these contributions to improve the order of accuracy of the solution and the associated gradients.

The context and scope of the present work is within this idea, which is applied to mixed formulations of the Poisson problem. Mixed formulations of the Poisson problem (e.g., the Darcy flow equations) encompass a wide range of applications in the areas of thermo-mechanics, heat transfer, geotechnical and petroleum engineering. We will consider both continuous Galerkin (CG) and discontinuous Galerkin (DG) formulations, to demonstrate the flexibility of the proposed approach. For the sake of simplicity, we focus on piecewise linear finite element approximations. The main contributions of this paper can be summarized as follows:

1. We elaborate on the enrichment approach used in $[35,31,32,33]$ in the context of body fitted stabilized finite elements in mixed form. We propose a symmetric enrichment method that can be rigorously proved to be stable and third-/second-order accurate for the primary variable/gradient, respectively.

2. We extend these enriched schemes to embedded computations using the SBM. Higher-order extrapolations are introduced to preserve the same consistency of the enriched formulations, thus providing a genuinely secondorder embedded method for both the primary variable and its gradient, and a third-order method for the primary variable when only Dirichlet boundary conditions are embedded.

3. We provide an extensive numerical investigation of the properties of the methods proposed, confirming the expected orders of accuracy and stability properties. In particular, we show that quadratic exact solutions can be recovered within machine precision with linear elements, and that additional stabilization terms are required with the non-symmetric enrichment, in order to obtain stability properties similar to those of the symmetric enrichment.

In conclusion, the proposed constructions allow to overcome one of the limitations of the original shifted boundary method, providing a genuinely high-order formulation. 
The present article is organized as follows: the strong formulation of the problem of interest is presented in Section 2; the variational formulations for conformal grids are given in Section 3; the shifted boundary method is presented in Section 4; Section 5 is devoted to the primary variable enrichment and the construction of numerical schemes of improved accuracy; finally, an extensive validation of the proposed methods is detailed in Section 6; and conclusions are summarized in Section 7.

\section{Problem statement: Darcy flow}

Darcy's flow equations provide a homogenized macroscopic transport model through porous media. The Darcy operator can also be interpreted as a mixed form of the Poisson operator, in which the gradient (i.e., the flux) is solved as a variable together with the primary variable (i.e., the pressure). Darcy-type operators are very important in a number of fields that do not necessarily involve flow in porous media, such as heat transfer for systems with rough distributions of conductivities, etc. The Darcy flow problem is governed by the following set of equations:

$$
\left\{\begin{aligned}
\boldsymbol{\Lambda}^{-1} \boldsymbol{\beta}+\boldsymbol{\nabla} p & =0 & & \text { in } \Omega \\
\boldsymbol{\nabla} \cdot \boldsymbol{\beta} & =\phi & & \text { in } \Omega \\
p & =p_{D} & & \text { on } \Gamma_{D} \\
\boldsymbol{\beta} \cdot \boldsymbol{n} & =h_{N} & & \text { on } \Gamma_{N}
\end{aligned}\right.
$$

where $\boldsymbol{\beta}$ denotes the flux, $p$ the pressure, $\boldsymbol{\Lambda}$ the permeability tensor and $\phi$ a source term. Note that the permeability is in general spatially dependent, that is $\boldsymbol{\Lambda}=\boldsymbol{\Lambda}(\boldsymbol{x})$, and may even be discontinuous. The boundary of the computational domain $\Omega$ is denoted $\partial \Omega$, and partitioned into $\Gamma_{D}$ and $\Gamma_{N}$, on which Dirichlet and Neumann boundary conditions are imposed, respectively. In particular, we have the following non-overlapping decomposition of the boundary: $\partial \Omega=\overline{\Gamma_{N} \cup \Gamma_{D}}, \Gamma_{N} \cap \Gamma_{D}=\emptyset$.

\section{Conformal mixed variational formulations}

We start our discussion by introducing the base variational formulations on conformal (i.e., body-fitted) grids, and for that purpose, by introducing some general notation.

\subsection{Generalities}

Let $\mathscr{T}_{h}$ denote a tessellation of the domain $\Omega$, composed of non-overlapping regular elements $K$, with $\mathscr{T}_{h}=\cup K$. For a given element $K$, we denote by $|K|$ its area, by $\partial K$ its boundary, by $\gamma_{K}$ a generic face/edge, and by $h_{K}$ its reference length. We denote the reference mesh size by $h=\max _{K \in \mathscr{T}_{h}} h_{K}$. The set of internal edges is denoted by $\mathscr{E}_{i}$, and for an interior edge $\gamma_{K}, K^{+}$and $K^{-}$are the elements on each side of it. For a given face/edge $\gamma_{K}$ of size $\left|\gamma_{K}\right|$, the characteristic length in a direction perpendicular to it is denoted by $h^{\perp}$, and computed as:

$$
h^{\perp}= \begin{cases}\frac{\left|K^{+}\right|+\left|K^{-}\right|}{2\left|\gamma_{K}\right|} & \text { if } \gamma_{K} \cap \partial \Omega=\emptyset \\ \frac{|K|}{2\left|\gamma_{K}\right|} & \text { if } \gamma_{K} \cap \partial \Omega \neq \emptyset\end{cases}
$$

The mesh is assumed to verify classical regularity assumptions. In particular it is assumed that there exist bounded constants $\mathscr{C}_{1}, \mathscr{C}_{2} \in \mathbb{R}^{+}$such that:

$$
\mathscr{C}_{1} h \leq h^{\perp} \leq \mathscr{C}_{2} h
$$

Let us now introduce the function spaces that will be used to build the discontinuous Galerkin (DG) and continuous Galerkin (CG) schemes. The discontinuous Galerkin spaces are defined as:

$$
\begin{aligned}
& S_{d}^{l}(\Omega)=\left\{p \in L^{2}(\Omega):\left.p\right|_{K} \in \mathscr{P}^{l}(K), \forall K \in \mathscr{T}_{h}\right\} \\
& \boldsymbol{V}_{d}^{k}(\Omega)=\left\{\boldsymbol{\beta} \in\left(L^{2}(\Omega)\right)^{d}, \boldsymbol{\nabla} \cdot \boldsymbol{\beta} \in L^{2}(\Omega):\left.\boldsymbol{\beta}\right|_{K} \in\left(\mathscr{P}^{k}(K)\right)^{d}, \forall K \in \mathscr{T}_{h}\right\}
\end{aligned}
$$


where $\mathscr{P}^{n}(K)$ is the space of polynomial functions of order at most $n$ on $K$. From these definitions, the continuous Galerkin spaces are defined as

$$
\begin{aligned}
& S_{c}^{l}(\Omega)=S_{d}^{l}(\Omega) \cap C^{0}(\Omega) \\
& \boldsymbol{V}_{c}^{k}(\Omega)=V_{d}^{k}(\Omega) \cap\left(C^{0}(\Omega)\right)^{d}
\end{aligned}
$$

where $C^{0}(\Omega)$ denotes the set of continuous functions on $\Omega$. We also introduce the tensor product spaces $\boldsymbol{M}_{d}(\Omega)=$ $\boldsymbol{V}_{d}^{k}(\Omega) \times S_{d}^{l}(\Omega)$ and $\boldsymbol{M}_{c}(\Omega)=\boldsymbol{V}_{c}^{k}(\Omega) \times S_{c}^{l}(\Omega)$. The $L^{2}(K)$ inner products over an element and its boundary are defined as:

$$
\begin{array}{rlrlrl}
(v, w)_{K} & =\int_{K} v w & \text { and } & & (\boldsymbol{v}, \boldsymbol{w})_{K} & =\int_{K} \boldsymbol{v} \cdot \boldsymbol{w} \\
\langle u, v\rangle_{\partial K} & -\int_{\partial K} u v & \text { and } & \langle\boldsymbol{u}, \boldsymbol{v}\rangle_{\partial K} & =\int_{K} \boldsymbol{u} \cdot \boldsymbol{v}
\end{array}
$$

Next, we introduce the average and jump operators $\{\cdot\}$ and $\llbracket \cdot \rrbracket$ for discontinuous scalar and vector fields as follows:

$$
\begin{array}{ll}
\{p\}=\frac{1}{2}\left(p^{+}+p^{-}\right) & \llbracket p \rrbracket=p^{+} \boldsymbol{n}^{+}+p^{-} \boldsymbol{n}^{-} \\
\{\boldsymbol{\beta}\}=\frac{1}{2}\left(\boldsymbol{\beta}^{+}+\boldsymbol{\beta}^{-}\right) & \llbracket \boldsymbol{\beta} \rrbracket=\boldsymbol{\beta}^{+} \cdot \boldsymbol{n}^{+}+\boldsymbol{\beta}^{-} \cdot \boldsymbol{n}^{-}
\end{array}
$$

where the + and - signs indicate the two sides of an edge/face. By a reordering argument, the following useful identity can be proved:

$$
\sum_{K}\langle w, \boldsymbol{v} \cdot \boldsymbol{n}\rangle_{\partial K}=\langle\{w\}, \llbracket \boldsymbol{v} \rrbracket\rangle_{\mathscr{E}_{i}}+\langle\llbracket w \rrbracket,\{\boldsymbol{v}\}\rangle_{\mathscr{E}_{i}}+\langle w, \boldsymbol{v} \cdot \boldsymbol{n}\rangle_{\partial \Omega}
$$

\subsection{Continuous and discontinuous Galerkin mixed formulations}

We briefly recall here the construction of discontinuous Galerkin scheme and refer to [12, 3, 13] and references therein for more details. The corresponding continuous formulations will be obtained by enforcing continuity of the discrete approximations across element interfaces.

Discontinuous Galerkin schemes have undergone considerable development from early works [42, 13, 12] and allow for a robust treatment of rough distributions of permeabilities/conductivities [3, 13, 21, 45, 14]. The main drawback of DG methods is the large number of degrees-of-freedom, which relates to the overall computational cost and has spurred the development of the hybridizable DG method [11], the enriched Galerkin approach [25], or the variational multiscale DG approach $[23,49]$ ). These methods are generally more complex to implement, and for this reason we also consider stabilized CG methods [30], which may become more appropriate and efficient when the variation of the permeabilities/conductivities is smooth.

Specifically, we consider the stabilized CG and DG schemes of Masud et. al. [30,22] and the interior penalty DG scheme of $[13,3]$. As a starting point, each equation of the Darcy problem (1) is multiplied by the test function pair $(\boldsymbol{w}, q) \in \boldsymbol{M}_{d}(\Omega)$ and the result is integrated over a generic element:

$$
\begin{aligned}
\left(\boldsymbol{w}, \boldsymbol{\Lambda}^{-1} \boldsymbol{\beta}\right)_{K}+(\boldsymbol{w}, \boldsymbol{\nabla} p)_{K} & =\left(\boldsymbol{w}, \boldsymbol{\Lambda}^{-1} \boldsymbol{\beta}\right)_{K}-(\boldsymbol{\nabla} \cdot \boldsymbol{w}, p)_{K}+\langle\boldsymbol{w} \cdot \boldsymbol{n}, p\rangle_{\partial K}=0 \\
(q, \boldsymbol{\nabla} \cdot \boldsymbol{\beta})_{K} & =-(\boldsymbol{\nabla} q, \boldsymbol{\beta})_{K}+\langle q, \boldsymbol{\beta} \cdot \boldsymbol{n}\rangle_{\partial K}=(q, \phi)_{K}
\end{aligned}
$$

The boundary traces are then replaced by the numerical fluxes $\hat{p}, \hat{\beta}$ and an integration by parts is performed again on the second equation before summing on all the elements of the mesh:

$$
\begin{aligned}
\left(\boldsymbol{w}, \boldsymbol{\Lambda}^{-1} \boldsymbol{\beta}\right)_{\Omega}-(\boldsymbol{\nabla} \cdot \boldsymbol{w}, p)_{\Omega}+\sum_{K}\langle\boldsymbol{w} \cdot \boldsymbol{n}, \hat{p}\rangle_{\partial K} & =0 \\
(q, \boldsymbol{\nabla} \cdot \boldsymbol{\beta})_{\Omega}+\sum_{K}\langle q,(\hat{\boldsymbol{\beta}}-\boldsymbol{\beta}) \cdot \boldsymbol{n}\rangle_{\partial K} & =(q, \phi)_{\Omega}
\end{aligned}
$$


The numerical fluxes link the discontinuous polynomial approximations of the solution between neighboring elements. Using the definition of average and jump operators along with equality (8), the set of equations (10) becomes:

$$
\begin{aligned}
\left(\boldsymbol{w}, \boldsymbol{\Lambda}^{-1} \boldsymbol{\beta}\right)_{\Omega}-(\boldsymbol{\nabla} \cdot \boldsymbol{w}, p)_{\Omega}+\langle\llbracket \boldsymbol{w} \rrbracket,\{\hat{p}\}\rangle_{\mathscr{E}_{i}}+\langle\{\boldsymbol{w}\}, \llbracket \hat{p} \rrbracket\rangle_{\mathscr{E}_{i}}+\langle\boldsymbol{w} \cdot \boldsymbol{n}, \hat{p}\rangle_{\partial \Omega} & =0 \\
(q, \boldsymbol{\nabla} \cdot \boldsymbol{\beta})_{\Omega}+\langle\llbracket q \rrbracket,\{\hat{\boldsymbol{\beta}}-\boldsymbol{\beta}\}\rangle_{\mathscr{E}_{i}}+\langle\{q\}, \llbracket \hat{\boldsymbol{\beta}}-\boldsymbol{\beta} \rrbracket\rangle_{\mathscr{E}_{i}}+\langle q,(\hat{\boldsymbol{\beta}}-\boldsymbol{\beta}) \cdot \boldsymbol{n}\rangle_{\partial \Omega} & =(q, \phi)_{\Omega}
\end{aligned}
$$

The definition of the numerical fluxes is what differentiates the many possible DG formulations. In this work, we use the Interior Penalty (IP) method [13, 50, 4, 3], and the numerical fluxes are defined as

$$
\left\{\begin{array}{llr}
\hat{p}=\{p\}+\alpha_{p} \llbracket \boldsymbol{\beta} \rrbracket, & \hat{\boldsymbol{\beta}}=\{\boldsymbol{\beta}\}+\alpha_{\beta} \llbracket p \rrbracket, & \text { on } \mathscr{E}_{i} \\
\hat{p}=p_{D}, & \hat{\boldsymbol{\beta}}=\boldsymbol{\beta}+\alpha_{\beta}\left(p-p_{D}\right) \boldsymbol{n}, & \text { on } \Gamma_{D} \\
\hat{p}=p, & \hat{\boldsymbol{\beta}} \cdot \boldsymbol{n}=h_{N}, & \text { on } \Gamma_{N}
\end{array}\right.
$$

where $\alpha_{p}=\tilde{\alpha}_{p} h^{\perp} /\|\boldsymbol{\Lambda}\|$ and $\alpha_{\beta}=\tilde{\alpha}_{\beta} /\left(h^{\perp}\right)\|\boldsymbol{\Lambda}\|$ are penalty coefficients, with $\tilde{\alpha}_{p}$ and $\tilde{\alpha}_{\beta}$ real positive constants. As proposed in [22], a stabilizing term similar to the one employed in the continuous formulation [30] can be added and the final formulation yields:

$$
\begin{aligned}
B_{d}(\boldsymbol{w}, q ; \boldsymbol{\beta}, p)= & B_{d}^{G a l}(\boldsymbol{w}, q ; \boldsymbol{\beta}, p)+B^{S t a b}(\boldsymbol{w}, q ; \boldsymbol{\beta}, p)=L_{d}^{G a l}(\boldsymbol{w}, q)+L^{S t a b}(\boldsymbol{w}, q) \\
B_{d}^{G a l}(\boldsymbol{w}, q ; \boldsymbol{\beta}, p)= & \left(\boldsymbol{w}, \boldsymbol{\Lambda}^{-1} \boldsymbol{\beta}\right)_{\Omega}-(\boldsymbol{\nabla} \cdot \boldsymbol{w}, p)_{\Omega}+(q, \boldsymbol{\nabla} \cdot \boldsymbol{\beta})_{\Omega}+\langle\llbracket \boldsymbol{w} \rrbracket,\{p\}\rangle_{\mathscr{E}_{i}}-\langle\{q\}, \llbracket \boldsymbol{\beta} \rrbracket\rangle_{\mathscr{E}_{i}}+\left\langle\llbracket q \rrbracket, \alpha_{\beta} \llbracket p \rrbracket\right\rangle_{\mathscr{E}_{i}} \\
& +\left\langle\llbracket \boldsymbol{w} \rrbracket, \alpha_{p} \llbracket \boldsymbol{\beta} \rrbracket\right\rangle_{\mathscr{E}_{i}}+\langle\boldsymbol{w} \cdot \boldsymbol{n}, p\rangle_{\Gamma_{N}}-\langle q, \boldsymbol{\beta} \cdot \boldsymbol{n}\rangle_{\Gamma_{N}}+\left\langle q, \alpha_{\beta} p\right\rangle_{\Gamma_{D}} \\
B^{S t a b}(\boldsymbol{w}, q ; \boldsymbol{\beta}, p)= & \frac{1}{2}\left(-\boldsymbol{\Lambda}^{-1} \boldsymbol{w}+\boldsymbol{\nabla} q, \boldsymbol{\beta}+\boldsymbol{\Lambda} \boldsymbol{\nabla} p\right)_{\Omega}+\frac{\zeta_{\text {div }}}{2}\left(\boldsymbol{\nabla} \cdot \boldsymbol{w},\|\boldsymbol{\Lambda}\| h^{2} \boldsymbol{\nabla} \cdot \boldsymbol{\beta}\right)_{\Omega} \\
L_{d}^{G a l}(\boldsymbol{w}, q)= & (q, \phi)_{\Omega}-\left\langle\boldsymbol{w} \cdot \boldsymbol{n}, p_{D}\right\rangle_{\Gamma_{D}}+\left\langle q, \alpha_{\beta} p_{D}\right\rangle_{\Gamma_{D}}-\left\langle q, h_{N}\right\rangle_{\Gamma_{N}} \\
L^{\text {Stab }}(\boldsymbol{w}, q)= & \frac{\zeta_{\text {div }}}{2}\left(\boldsymbol{\nabla} \cdot \boldsymbol{w},\|\boldsymbol{\Lambda}\| h^{2} \phi\right)_{\Omega}
\end{aligned}
$$

where $\zeta_{\text {div }}=\mathscr{O}(1)$ is a stabilization constant. In the stabilization $B^{S t a b}(\boldsymbol{w}, q ; \boldsymbol{\beta}, p)$, the first term containing the residual of the momentum equation $\boldsymbol{\beta}+\boldsymbol{\Lambda} \boldsymbol{\nabla} p$ will be referred as "momentum stabilization" while the term involving the divergence of the flux will be referred as "div-div" stabilization. From (13), the continuous stabilized formulation can be obtained by removing terms containing jumps across internal edges/faces, and by replacing averages of the unknowns and test functions by their unique continuous values. The stability of the proposed formulation was proved in $[22,30]$ in the norm:

$$
\begin{aligned}
\|(\boldsymbol{\beta}, p)\|^{2}= & \frac{1}{2}\left(\left\|\boldsymbol{\Lambda}^{-\mathbf{1} / \mathbf{2}} \boldsymbol{\beta}\right\|_{\Omega}^{2}+\left\|\boldsymbol{\Lambda}^{\mathbf{1} / \mathbf{2}} \boldsymbol{\nabla} p\right\|_{\Omega}^{2}\right)+\frac{\zeta_{\mathrm{div}}}{2}\left\|\boldsymbol{\Lambda}^{1 / 2} h \boldsymbol{\nabla} \cdot \boldsymbol{\beta}\right\|_{\Omega}^{2}+\left\|\left(\tilde{\alpha}_{\beta}\|\boldsymbol{\Lambda}\| / h^{\perp}\right)^{1 / 2} p\right\|_{\Gamma_{D}}^{2} \\
& +\left\|\left(\tilde{\alpha}_{\beta}\|\boldsymbol{\Lambda}\| / h^{\perp}\right)^{1 / 2} \llbracket p \rrbracket\right\|_{\mathscr{E}_{i}}^{2}+\left\|\left(\tilde{\alpha}_{p} h^{\perp} /\|\boldsymbol{\Lambda}\|\right)^{1 / 2} \llbracket \boldsymbol{\beta} \rrbracket\right\|_{\mathscr{E}_{i}}^{2}
\end{aligned}
$$

with the following error estimate:

$$
\left\|\left.\left|\boldsymbol{\beta}_{e}-\boldsymbol{\beta}, p_{e}-p\|\| \leq C_{1} h^{k+1}\right| \boldsymbol{\beta}\right|_{k+1}+C_{2} h^{l}|p|_{l+1}\right.
$$

where $C_{1}$ and $C_{2}$ are two constants. Hence this method converges with order $r=\min (k+1, l)$ for the flux. $L^{2}$-error estimates derived in [5] show that the pressure converges with order $r+1$. For linear approximations, $k=l=1$, meaning that the pressure is second-order accurate and the flux is first-order accurate, as will also be verified in Section 6.

Remark 1. The definition of the numerical fluxes (12) can be related to the variational multiscale approach proposed by Badia and Codina [5].

\section{Shifted boundary methods in mixed form}

\subsection{General notations, the true and surrogate domains}

Let us consider now an embedded discretization, in which the computational grid does not conform to the boundary (see Figure 1). We introduce the notion of a surrogate boundary $\tilde{\Gamma}$, composed of the faces/edges of the mesh that are 
the closest to the true boundary $\Gamma$, in some sense. $\tilde{\Gamma}$ can be constructed, for example, by computing the intersections of the grid and the true boundary $\Gamma$ and using closest-point projection algorithms to detect the closest face/edge of $\tilde{\Gamma}$ to $\Gamma$. Other choices are of course possible, such as level-set functions, for instance, as long as the overall topology of $\Gamma$ and $\tilde{\Gamma}$ are close to each other, that is if $\Gamma$ has a certain number of holes, the same number of holes needs to be also present in $\tilde{\Gamma}$. The surrogate boundary $\tilde{\Gamma}$ encloses the surrogate domain $\tilde{\Omega}$. In particular, $\tilde{\boldsymbol{n}}$ indicates the unit outward normal of $\tilde{\Gamma}$, to be distinguished from the outward normal $\boldsymbol{n}$ of $\Gamma$. The mapping from the surrogate to true interface is then defined as:

$$
\begin{aligned}
\boldsymbol{M}: \tilde{\Gamma} & \rightarrow \Gamma \\
\tilde{\boldsymbol{x}} & \mapsto \boldsymbol{x}
\end{aligned}
$$

In particular, the map $\boldsymbol{M}$ can be characterized through a distance vector function:

$$
\boldsymbol{d}_{M}(\tilde{\boldsymbol{x}})=\boldsymbol{x}-\tilde{\boldsymbol{x}}=[\boldsymbol{M}-\boldsymbol{I}](\tilde{\boldsymbol{x}})
$$

In the following, we will simply define the distance vector function as $\boldsymbol{d}$, and note the following relation with the normal of the true boundary:

$$
n=\frac{d}{\|d\|}
$$

We recall here an assumption of sufficient geometric resolution, discussed in detail in the original development of the SBM [28]:

Assumption 1. The vector distance $\boldsymbol{d}$ is defined as $\boldsymbol{d}=\|\boldsymbol{d}\| \boldsymbol{n}$, where the normal $\boldsymbol{n}$ to the true boundary and the normal $\tilde{\boldsymbol{n}}$ to the surrogate boundary satisfy:

$$
\boldsymbol{n} \cdot \tilde{\boldsymbol{n}}>0
$$

Remark 2. Condition (20) is a resolution condition on the grid used in SB simulations. In practice, a check is performed at the beginning of every simulation to verify that (20) is satisfied. If this is not the case, the grid is refined (for example, splitting every edge of the grid into two), and (20) is checked again. This simple strategy proved extremely robust in ensuring the well-posedness of the overall SB approach. In principle, more sophisticated edge swapping strategies can be used to ensure the grid satisfies (20), although we did not perform numerical experiments to confirm these claims. There is also the more general question of the existence of the distance function between the surrogate and true boundary. This is a more fundamental issue common to all immersed/embedded methods and not just the SBM. For a more detailed view on the minimal conditions of existence, the reader is prompted to read [41]. Condition (20) is somewhat related to the conditions specified in [41], in the sense that it is a sufficient condition (although possibly not necessary) for the well-posedness of the existence of a distance function.

Through the map $\boldsymbol{M}$, it is possible to define the extension $\bar{\psi}$ on $\tilde{\Gamma}$ of a function $\psi$ originally defined on $\Gamma$, as

$$
\bar{\psi}(\tilde{\boldsymbol{x}}) \equiv \psi(\boldsymbol{M}(\tilde{\boldsymbol{x}}))
$$

For instance, the unit normal $\boldsymbol{n}$ and tangential vectors $\boldsymbol{\tau}_{i}\left(1<i<n_{d}-1\right)$ of the true boundary $\Gamma$ can be extended to the boundary $\tilde{\Gamma}$ as follows:

$$
\begin{aligned}
\overline{\boldsymbol{n}}(\tilde{\boldsymbol{x}}) & \equiv \boldsymbol{n}(\boldsymbol{M}(\tilde{\boldsymbol{x}})) \\
\overline{\boldsymbol{\tau}}_{i}(\tilde{\boldsymbol{x}}) & \equiv \boldsymbol{\tau}_{i}(\boldsymbol{M}(\tilde{\boldsymbol{x}}))
\end{aligned}
$$

In what follows, and for the sake of simplicity, we will omit the bar in the expressions of the extensions of the normal and tangent vectors whenever there is no possibility of confusion, thus writing $\boldsymbol{n}(\tilde{\boldsymbol{x}})$ and $\boldsymbol{\tau}_{i}(\tilde{\boldsymbol{x}})$. We can also introduce the derivatives in the directions $\boldsymbol{n}$ and $\boldsymbol{\tau}_{i}$ of a function $\psi$ at a point $\tilde{\boldsymbol{x}} \in \tilde{\Gamma}$ :

$$
\begin{aligned}
\psi_{, n}(\tilde{\boldsymbol{x}}) & =\boldsymbol{\nabla} \psi(\tilde{\boldsymbol{x}}) \cdot \boldsymbol{n}(\tilde{\boldsymbol{x}}) \\
\psi_{, \tau_{i}}(\tilde{\boldsymbol{x}}) & =\boldsymbol{\nabla} \psi(\tilde{\boldsymbol{x}}) \cdot \boldsymbol{\tau}_{i}(\tilde{\boldsymbol{x}})
\end{aligned}
$$




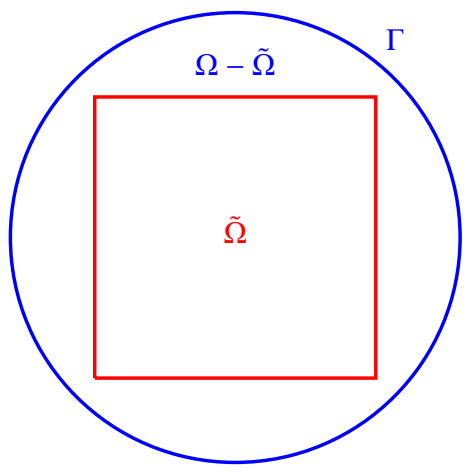

(a) The surrogate domain $\tilde{\Omega}$ and the true domain $\Omega$

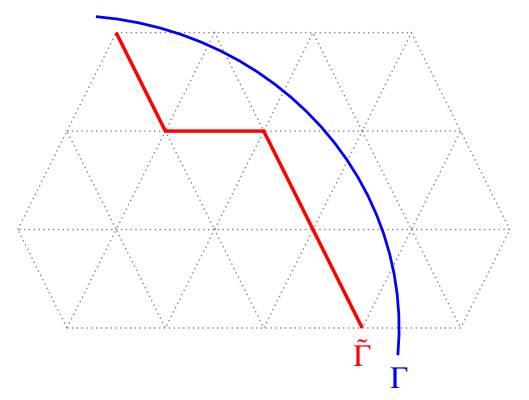

(b) The surrogate boundary $\tilde{\Gamma}$ and the true boundary $\Gamma$

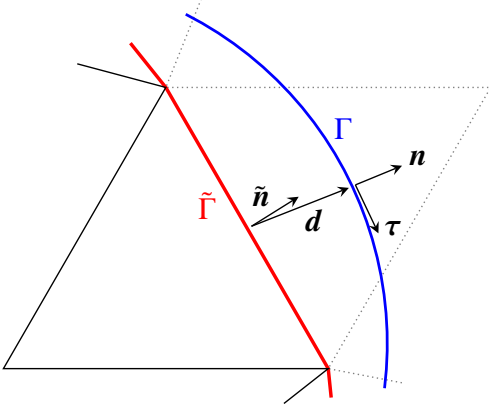

(c) The distance vector $\boldsymbol{d}$, the tangent $\boldsymbol{\tau}$ and normal $\boldsymbol{n}$ to the true boundary, and the normal $\tilde{\boldsymbol{n}}$ to the surrogate boundary

Figure 1: Surrogate and true domain/boundary notations.

These constructions are particularly useful if we consider the solution $u$ to a partial differential equation and, for example, we desire to extend the Dirichlet boundary condition $u_{D}$ from the true boundary $\Gamma_{D}$ to a surrogate boundary $\tilde{\Gamma}_{D}$. In particular, the following Taylor expansion centered at $\tilde{\boldsymbol{x}} \in \tilde{\Gamma}_{D}$ holds for $\boldsymbol{x}=\boldsymbol{M}(\tilde{\boldsymbol{x}})=\tilde{\boldsymbol{x}}+\boldsymbol{d}(\tilde{\boldsymbol{x}}) \in \Gamma_{D}$ :

$$
\begin{aligned}
u_{D}(\boldsymbol{x}) & =u_{D}(\tilde{\boldsymbol{x}}+\boldsymbol{d}(\tilde{\boldsymbol{x}})) \\
& =u_{D}(\tilde{\boldsymbol{x}})+\boldsymbol{\nabla} u(\tilde{\boldsymbol{x}}) \cdot \boldsymbol{d}(\tilde{\boldsymbol{x}})+\frac{1}{2} \boldsymbol{d}^{T}(\tilde{\boldsymbol{x}}) \mathscr{H}(u) \boldsymbol{d}(\tilde{\boldsymbol{x}})+\mathscr{O}\left(\|\boldsymbol{d}(\tilde{\boldsymbol{x}})\|^{3}\right)
\end{aligned}
$$

where $\mathscr{H}(u)$ denotes the Hessian of $u$. This last expression can be used to develop a new strategy for the imposition of Dirichlet boundary conditions in the context of embedded methods. Depending on the desired accuracy, additional terms can be considered in the Taylor expansion.

\subsection{The shifted boundary variational formulation}

We now propose to extend the scheme (13) to an embedded formulation, using the SBM [28, 29]. Looking at equations (11), the integrations over internal edges are not impacted by the integration on the surrogate domain. However, the boundary integral on the surrogate domain $\partial \tilde{\Omega}$ now involves the surrogate normal $\tilde{\boldsymbol{n}}$. Thus, equations (11) are written as

$$
\begin{aligned}
& \left(\boldsymbol{w}, \boldsymbol{\Lambda}^{-1} \boldsymbol{\beta}\right)_{\tilde{\Omega}}-(\boldsymbol{\nabla} \cdot \boldsymbol{w}, p)_{\tilde{\Omega}}+\langle\llbracket \boldsymbol{w} \rrbracket,\{\hat{p}\}\rangle_{\mathscr{E}_{i}}+\langle\{\boldsymbol{w}\}, \llbracket \hat{p} \rrbracket\rangle_{\mathscr{E}_{i}}+\langle\boldsymbol{w} \cdot \tilde{\boldsymbol{n}}, \hat{p}\rangle_{\partial \tilde{\Omega}}=0 \\
& (q, \boldsymbol{\nabla} \cdot \boldsymbol{\beta})_{\tilde{\Omega}}+\langle\llbracket q \rrbracket,\{\hat{\boldsymbol{\beta}}-\boldsymbol{\beta}\}\rangle_{\mathscr{E}_{i}}+\langle\{q\}, \llbracket \hat{\boldsymbol{\beta}}-\boldsymbol{\beta} \rrbracket\rangle_{\mathscr{E}_{i}}+\langle q,(\hat{\boldsymbol{\beta}}-\boldsymbol{\beta}) \cdot \tilde{\boldsymbol{n}}\rangle_{\partial \tilde{\Omega}}=(q, \phi)_{\tilde{\Omega}}
\end{aligned}
$$

The definitions of the numerical fluxes (12) need to be modified accordingly on the boundaries. The main differences arise for Neumann boundary conditions, which involve the flux component along the normal to the true boundary. The first step is to decompose the surrogate normal $\tilde{\boldsymbol{n}}$ into the normal $\boldsymbol{n}$ and tangents $\boldsymbol{\tau}_{i}$ to the true boundary. Then, the boundary term in equation (26) yields, on Neumann boundaries,

$$
\langle q,(\hat{\boldsymbol{\beta}}-\boldsymbol{\beta}) \cdot \tilde{\boldsymbol{n}}\rangle_{\tilde{\Gamma}_{N}}=\langle q(\boldsymbol{n} \cdot \tilde{\boldsymbol{n}}),(\hat{\boldsymbol{\beta}}-\boldsymbol{\beta}) \cdot \boldsymbol{n}\rangle_{\tilde{\Gamma}_{N}}+\left\langle q\left(\boldsymbol{\tau}_{i} \cdot \tilde{\boldsymbol{n}}\right),(\hat{\boldsymbol{\beta}}-\boldsymbol{\beta}) \cdot \boldsymbol{\tau}_{i}\right\rangle_{\tilde{\Gamma}_{N}}
$$

In addition, we impose the following condition on the tangential flux:

$$
\hat{\boldsymbol{\beta}} \cdot \boldsymbol{\tau}_{i}=\boldsymbol{\beta} \cdot \boldsymbol{\tau}_{i} \text {, on } \tilde{\Gamma}_{N}
$$

Then, omitting the stabilization terms, the scheme (13) reads:

$$
\begin{aligned}
& \left(\boldsymbol{w}, \boldsymbol{\Lambda}^{-1} \boldsymbol{\beta}\right)_{\tilde{\Omega}}-(\boldsymbol{\nabla} \cdot \boldsymbol{w}, p)_{\tilde{\Omega}}+(q, \boldsymbol{\nabla} \cdot \boldsymbol{\beta})_{\tilde{\Omega}}+\langle\llbracket \boldsymbol{w} \rrbracket,\{p\}\rangle_{\mathscr{E}_{i}}-\langle\{q\}, \llbracket \boldsymbol{\beta} \rrbracket\rangle_{\mathscr{E}_{i}}+\left\langle\llbracket q \rrbracket, \alpha_{\beta} \llbracket p \rrbracket\right\rangle_{\mathscr{E}_{i}}+\left\langle\llbracket \boldsymbol{w} \rrbracket, \alpha_{p} \llbracket \boldsymbol{\beta} \rrbracket\right\rangle_{\mathscr{E}_{i}} \\
& \left.+\langle\boldsymbol{w} \cdot \tilde{\boldsymbol{n}}, p\rangle_{\tilde{\Gamma}_{N}}-\left\langle q(\boldsymbol{n} \cdot \tilde{\boldsymbol{n}}), \boldsymbol{\beta} \cdot \boldsymbol{n}-\tilde{h}_{N}\right)\right\rangle_{\tilde{\Gamma}_{N}}+\left\langle\boldsymbol{w} \cdot \tilde{\boldsymbol{n}}, \tilde{p}_{D}\right\rangle_{\tilde{\Gamma}_{D}}+\left\langle q, \alpha_{\beta}\left(p-\tilde{p}_{D}\right)\right\rangle_{\tilde{\Gamma}_{D}}=0
\end{aligned}
$$

Here we wrote $\tilde{p}_{D}$ and $\tilde{h}_{N}$ to indicate the fact that these values are not equal to $p_{D}$ and $h_{N}$, which are instead defined on the true boundaries $\Gamma_{D}$ and $\Gamma_{N} . \tilde{p}_{D}$ and $\tilde{h}_{N}$ need to be defined appropriately to preserve the accuracy of the schemes. 


\subsubsection{Shifted Dirichlet boundary conditions}

Following the methodology developed in [28], with the theory and notations proposed in Section 4, the Taylor expansion (24) is employed to extend the known function $p_{D}$ from the true boundaries $\Gamma_{D}$ onto the surrogate ones $\tilde{\Gamma}_{D}$ :

$$
p_{D}(\boldsymbol{x})=\bar{p}_{D}(\tilde{\boldsymbol{x}})=\tilde{p}_{D}(\tilde{\boldsymbol{x}})+\boldsymbol{\nabla} p \cdot \boldsymbol{d}+\mathscr{O}\left(\|\boldsymbol{d}\|^{2}\right)
$$

Thus, on Dirichlet boundaries, $\tilde{p}_{D}(\tilde{\boldsymbol{x}})=p_{D}-\boldsymbol{\nabla} p \cdot \boldsymbol{d}$ needs to be substituted in (12) in place of $p_{D}$.

\subsubsection{Shifted Neumann boundary condition}

Similar to the case of Dirichlet boundary conditions, on Neumann boundaries we have:

$$
h_{N}(\boldsymbol{x})=\bar{h}_{N}(\tilde{\boldsymbol{x}})=\tilde{h}_{N}(\tilde{\boldsymbol{x}})+[(\boldsymbol{\nabla} \boldsymbol{\beta}) \boldsymbol{d}] \cdot \boldsymbol{n}+\mathscr{O}\left(\|\boldsymbol{d}\|^{2}\right)
$$

so that $\tilde{h}_{N}(\tilde{\boldsymbol{x}})=h_{N}(\boldsymbol{x})-[(\boldsymbol{\nabla} \boldsymbol{\beta}) \boldsymbol{d}] \cdot \boldsymbol{n}$ needs to be substituted in (12) in place of $h_{N}$.

\subsubsection{Shifted Boundary Method scheme}

Using now the corrected (shifted) values of the boundary conditions (30)-(31), the final discontinuous weak formulation reads:

$$
\begin{aligned}
B_{d}(\boldsymbol{w}, q ; \boldsymbol{\beta}, p)= & B_{d}^{G a l}(\boldsymbol{w}, q ; \boldsymbol{\beta}, p)+B^{S t a b}(\boldsymbol{w}, q ; \boldsymbol{\beta}, p)=L_{d}^{G a l}(\boldsymbol{w}, q)+L^{S t a b}(\boldsymbol{w}, q) \\
B_{d}^{G a l}(\boldsymbol{w}, q ; \boldsymbol{\beta}, p)= & \left(\boldsymbol{w}, \boldsymbol{\Lambda}^{-1} \boldsymbol{\beta}\right)_{\tilde{\Omega}}-(\boldsymbol{\nabla} \cdot \boldsymbol{w}, p)_{\tilde{\Omega}}+(q, \boldsymbol{\nabla} \cdot \boldsymbol{\beta})_{\tilde{\Omega}}+\langle\llbracket \boldsymbol{w} \rrbracket,\{p\}\rangle_{\mathscr{E}_{i}}-\langle\{q\}, \llbracket \boldsymbol{\beta} \rrbracket\rangle_{\mathscr{E}_{i}}+\left\langle\llbracket q \rrbracket, \alpha_{\beta} \llbracket p \rrbracket\right\rangle_{\mathscr{E}_{i}} \\
& +\left\langle\llbracket \boldsymbol{w} \rrbracket, \alpha_{p} \llbracket \boldsymbol{\beta} \rrbracket\right\rangle_{\mathscr{E}_{i}}+\langle\boldsymbol{w} \cdot \tilde{\boldsymbol{n}}, p\rangle_{\tilde{\Gamma}_{N}}-\langle q(\boldsymbol{n} \cdot \tilde{\boldsymbol{n}}),(\boldsymbol{\beta}+[\boldsymbol{\nabla} \boldsymbol{\beta}] \boldsymbol{d}) \cdot \boldsymbol{n}\rangle_{\tilde{\Gamma}_{N}}-\langle\boldsymbol{w} \cdot \tilde{\boldsymbol{n}}, \boldsymbol{\nabla} p \cdot \boldsymbol{d}\rangle_{\tilde{\Gamma}_{D}} \\
& +\left\langle q+\boldsymbol{\nabla} q \cdot \boldsymbol{d}, \alpha_{\beta}(p+\boldsymbol{\nabla} p \cdot \boldsymbol{d})\right\rangle_{\tilde{\Gamma}_{D}} \\
L_{d}^{G a l}(\boldsymbol{w}, q)= & (q, \phi)_{\tilde{\Omega}}-\left\langle\boldsymbol{w} \cdot \tilde{\boldsymbol{n}}, \bar{p}_{D}\right\rangle_{\tilde{\Gamma}_{D}}+\left\langle q+\boldsymbol{\nabla} q \cdot \boldsymbol{d}, \alpha \bar{p}_{D}\right\rangle_{\tilde{\Gamma}_{D}}-\left\langle q(\boldsymbol{n} \cdot \tilde{\boldsymbol{n}}), \bar{h}_{N}\right\rangle_{\tilde{\Gamma}_{N}}
\end{aligned}
$$

Once again, the continuous stabilized variational formulation is obtained from (32) by neglecting all the internal jump terms:

$$
\begin{aligned}
B_{c}(\boldsymbol{w}, q ; \boldsymbol{\beta}, p)= & B_{c}^{G a l}(\boldsymbol{w}, q ; \boldsymbol{\beta}, p)+B^{S t a b}(\boldsymbol{w}, q ; \boldsymbol{\beta}, q)=L_{c}^{G a l}(\boldsymbol{w}, q)+L^{S t a b}(\boldsymbol{w}, q) \\
B_{c}^{G a l}(\boldsymbol{w}, q ; \boldsymbol{\beta}, p)= & \left(\boldsymbol{w}, \boldsymbol{\Lambda}^{-1} \boldsymbol{\beta}\right)_{\tilde{\Omega}}-(\boldsymbol{\nabla} \cdot \boldsymbol{w}, p)_{\tilde{\Omega}}+(q, \boldsymbol{\nabla} \cdot \boldsymbol{\beta})_{\tilde{\Omega}}+\langle\boldsymbol{w} \cdot \tilde{\boldsymbol{n}}, p\rangle_{\tilde{\Gamma}_{N}}-\langle q(\boldsymbol{n} \cdot \tilde{\boldsymbol{n}}),(\boldsymbol{\beta}+[\boldsymbol{\nabla} \boldsymbol{\beta}] \boldsymbol{d}) \cdot \boldsymbol{n}\rangle_{\tilde{\Gamma}_{N}} \\
& +\langle\boldsymbol{w} \cdot \tilde{\boldsymbol{n}}, \boldsymbol{\nabla} p \cdot \boldsymbol{d}\rangle_{\tilde{\Gamma}_{D}}+\left\langle q+\boldsymbol{\nabla} \cdot \boldsymbol{d}, \alpha_{\beta}(p+\boldsymbol{\nabla} p \cdot \boldsymbol{d})\right\rangle_{\tilde{\Gamma}_{D}} \\
L_{c}^{G a l}(\boldsymbol{w}, q)= & (q, \phi)_{\tilde{\Omega}}-\left\langle\boldsymbol{w} \cdot \tilde{\boldsymbol{n}}, \bar{p}_{D}\right\rangle_{\tilde{\Gamma}_{D}}+\left\langle q+\boldsymbol{\nabla} q \cdot \boldsymbol{d}, \alpha_{\beta} \bar{p}_{D}\right\rangle_{\tilde{\Gamma}_{D}}-\left\langle q(\boldsymbol{n} \cdot \tilde{\boldsymbol{n}}), \bar{h}_{N}\right\rangle_{\tilde{\Gamma}_{N}}
\end{aligned}
$$

From this continuous Galerkin formulation (33), the Euler-Lagrange equations can be obtained by performing integration by parts. The resulting expression is:

$$
\begin{aligned}
0= & \left(\boldsymbol{w},\left(\boldsymbol{\Lambda}^{-1} \boldsymbol{\beta}+\boldsymbol{\nabla} p\right)\right)_{\tilde{\Omega}}+(q,(\boldsymbol{\nabla} \cdot \boldsymbol{\beta}-\phi))_{\tilde{\Omega}} \\
& -\left\langle\boldsymbol{w} \cdot \tilde{\boldsymbol{n}}+\alpha_{\beta}(q+\boldsymbol{\nabla} q \cdot \boldsymbol{d}), p+\boldsymbol{\nabla} p \cdot \boldsymbol{d}-\bar{p}_{D}\right\rangle_{\tilde{\Gamma}_{D}} \\
& -\left\langle q(\boldsymbol{n} \cdot \tilde{\boldsymbol{n}}),(\boldsymbol{\beta}+(\boldsymbol{\nabla} \boldsymbol{\beta}) \boldsymbol{d}) \cdot \boldsymbol{n}-\bar{h}_{N}\right\rangle_{\tilde{\Gamma}_{N}}
\end{aligned}
$$

The Euler-Lagrange equations identify the consistency conditions enforced by the SBM: the partial differential equation is enforced on the interior of the surrogate domain $\tilde{\Omega}$; Dirichlet conditions for the pressure are approximately enforced by means of the Taylor expansion, in a Nitsche fashion [37]; and Neumann boundary conditions are also approximately enforced using the Taylor expansion.

As illustrated later in Section 6, the formulation (32) yields a first-order accurate flux, and a second-order accurate pressure for Dirichlet boundary conditions. The accuracy of the latter reduces to first order when embedded Neumann conditions are considered.

The next section is devoted to improving the formulation just described, so that second-order accuracy can be obtained for both pressure and flux, regardless of the type of embedded boundary condition. 


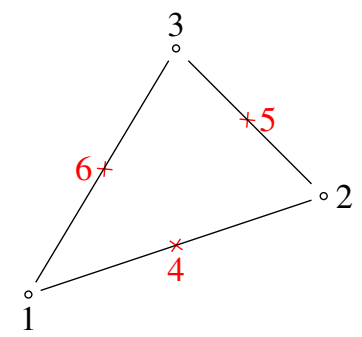

Figure 2: A triangle and the additional degrees-of-freedom for pressure enrichment (indicated with crosses).

\section{Pressure enrichment and high-order flux finite element scheme}

We propose next to modify the variational formulations (13) and (32) with the aim of obtaining at least secondorder accuracy on both pressure and flux for any kind of embedded boundary conditions. Taking inspiration from Caraeni [9], Nishikawa [35] and Mazaheri [31] improved the accuracy of advection-diffusion problems by using the mixed form of the equations. In particular, by exploiting the relations between the flux and the pressure gradients in the Darcy equation, we can construct a quadratic pressure approximation, as discussed in the following section.

\subsection{Enriched pressure approximation}

Our aim is to to define a quadratic pressure polynomial in each element. One way to achieve this is to add additional degrees-of-freedom associated to the pressure values at edge midpoints, as illustrated in Figure 2 (for simplicity, in two dimensions). These additional degrees-of-freedom become unknowns of the problem and thus the size of the algebraic system of equations to be solved increases. The resulting approximation $u_{h}$ of the variable $u$ reads:

$$
u_{h}(\boldsymbol{x})=\sum_{i, \text { node }} u_{i} \varphi_{i}^{\mathscr{P}^{2}}(\boldsymbol{x})+\sum_{j, \text { edge }} u_{j} \varphi_{j}^{\mathscr{P}^{2}}(\boldsymbol{x})
$$

where the $u_{i}$ and $u_{j}$ correspond to solution values associated to the nodal degrees-of-freedom (vertices), and to the extra $\mathscr{P}^{2}$ degrees-of-freedom (edge mid-points). In addition, we denote by $\varphi_{i}^{\mathscr{P}^{1}}$ and $\varphi_{i}^{\mathscr{P}^{2}}$ the linear and quadratic local shape functions.

Both in two and three space dimensions one can easily prove the following relations, which will be useful later on (cf. e.g. [16]):

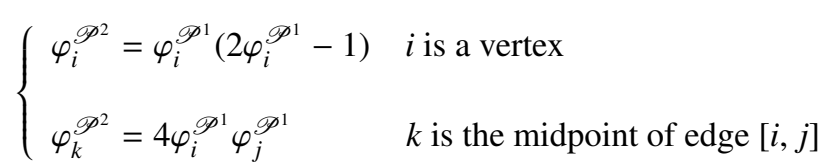

The idea is now to replace the mid-point unknowns by values appropriately defined using the known nodal values of the pressure and its gradient. For this purpose, Taylor expansions are used in combination with the mixed form of the equations. Looking at Figure 2, focusing on and edge $[i, j]$, the aim is to define the pressure at the midpoint $l$ using only information stored at nodes $i$ and $j$. We thus consider a third-order truncated Taylor expansion of the pressure along the edge, as well as a second-order expansion of the flux:

$$
\begin{aligned}
& p(\boldsymbol{x}+\boldsymbol{\delta} \boldsymbol{x})=p(\boldsymbol{x})+\boldsymbol{\nabla} p(\boldsymbol{x}) \cdot \boldsymbol{\delta} \boldsymbol{x}+\frac{1}{2} \boldsymbol{\delta} \boldsymbol{x}^{T} \mathscr{H}(p) \boldsymbol{\delta} \boldsymbol{x}+\mathscr{O}\left(\|\boldsymbol{\delta} \boldsymbol{x}\|^{3}\right) \\
& \boldsymbol{\beta}(\boldsymbol{x}+\boldsymbol{\delta} \boldsymbol{x})=\boldsymbol{\beta}(\boldsymbol{x})+(\boldsymbol{\nabla} \boldsymbol{\beta}(\boldsymbol{x})) \boldsymbol{\delta} \boldsymbol{x}+\mathscr{O}\left(\|\boldsymbol{\delta} \boldsymbol{x}\|^{2}\right)
\end{aligned}
$$

where $\mathscr{H}$ denotes the Hessian of the pressure.

Remark 3. In this section, the use of the Taylor expansion is not related to the SBM. In equation (37), $\boldsymbol{\delta} \boldsymbol{x}$ is defined along the edges of the grid, and not along the vector distance $\boldsymbol{d}$, introduced in Section 4 to map from the true to the surrogate boundary. 
If one considers (37) in combination with linear approximation, gradients are constant and the Hessian of the pressure is null. However, using the Darcy equation, the gradient of the pressure can be evaluated as $\boldsymbol{\nabla} p=-\boldsymbol{\Lambda}^{-1} \boldsymbol{\beta}$ which is actually linear and thus the Hessian can be now evaluated as

$$
\mathscr{H}(p)=-\left[\boldsymbol{\nabla}\left(\boldsymbol{\Lambda}^{-1} \boldsymbol{\beta}\right)+\boldsymbol{\nabla}\left(\boldsymbol{\Lambda}^{-1} \boldsymbol{\beta}\right)^{T}\right]
$$

In the last expression, the superscript ${ }^{T}$ denotes the transpose of a tensor. Hence,

$$
\boldsymbol{\delta} \boldsymbol{x}^{T} \mathscr{H}(p) \boldsymbol{\delta} \boldsymbol{x}=-\boldsymbol{\delta} \boldsymbol{x}^{T} \boldsymbol{\nabla}\left(\boldsymbol{\Lambda}^{-1} \boldsymbol{\beta}\right) \boldsymbol{\delta} \boldsymbol{x}
$$

and the Taylor expansions (37) can thus be rewritten as:

$$
\begin{aligned}
p(\boldsymbol{x}+\boldsymbol{\delta} \boldsymbol{x}) & \approx p(\boldsymbol{x})-\boldsymbol{\Lambda}^{-1} \boldsymbol{\beta} \cdot \boldsymbol{\delta} \boldsymbol{x}-\frac{1}{2} \delta \boldsymbol{x}^{T} \boldsymbol{\nabla}\left(\boldsymbol{\Lambda}^{-1} \boldsymbol{\beta}\right) \boldsymbol{\delta} \boldsymbol{x} \\
\left(\boldsymbol{\Lambda}^{-1} \boldsymbol{\beta}\right)(\boldsymbol{x}+\boldsymbol{\delta} \boldsymbol{x}) & \approx\left(\boldsymbol{\Lambda}^{-1} \boldsymbol{\beta}\right)(\boldsymbol{x})+\boldsymbol{\nabla}\left(\boldsymbol{\Lambda}^{-1} \boldsymbol{\beta}\right)(\boldsymbol{x}) \boldsymbol{\delta} \boldsymbol{x}
\end{aligned}
$$

Denoting $\boldsymbol{e}_{i \rightarrow j}=\boldsymbol{x}_{j}-\boldsymbol{x}_{i}$ the edge vector, the Taylor expansion of $\boldsymbol{\Lambda}^{-1} \boldsymbol{\beta}$ on the entire edge gives:

$$
\boldsymbol{\nabla}\left(\Lambda^{-1} \boldsymbol{\beta}\right)(x) \boldsymbol{e}_{i \rightarrow j}=\Lambda_{j}^{-1} \boldsymbol{\beta}_{j}-\Lambda_{i}^{-1} \boldsymbol{\beta}_{i}
$$

Remark 4. The error of the the Taylor expansion (37) can be estimated using the Lagrange formula of the remainder. In particular, classical arguments can be used to show that $\exists \boldsymbol{c} \in \boldsymbol{e}_{i \rightarrow j}$ such that:

$$
\left(\Lambda_{j}^{-1} \boldsymbol{\beta}_{j}-\Lambda_{i}^{-1} \boldsymbol{\beta}_{i}\right)-\boldsymbol{\nabla}\left(\Lambda^{-1} \boldsymbol{\beta}\right)(x) \boldsymbol{e}_{i \rightarrow j}=\left(\Lambda_{j}^{-1} \boldsymbol{\beta}_{j}-\Lambda_{i}^{-\mathbf{1}} \boldsymbol{\beta}_{i}\right)+\mathscr{H}(p)(x) \boldsymbol{e}_{i \rightarrow j}=\frac{1}{2 \times 2 !} \boldsymbol{e}_{i \rightarrow j}^{T} \nabla^{2} \boldsymbol{\beta}(c) \boldsymbol{e}_{i \rightarrow j}
$$

Applying the expansion at both node $i$ and $j$, and, for symmetry reasons, taking the average, we end up with the following estimate for the edge mid-point values of the pressure:

$$
p_{l}=\bar{p}^{e}+\frac{1}{8}\left(\boldsymbol{\Lambda}_{j}^{-1} \boldsymbol{\beta}_{j}-\Lambda_{i}^{-1} \boldsymbol{\beta}_{i}\right) \cdot\left(\boldsymbol{x}_{j}-\boldsymbol{x}_{i}\right)
$$

where $\bar{p}^{e}=\frac{1}{2}\left(p_{j}+p_{i}\right)$ is the average pressure on the edge. Feeding this expression back into (35) and using (36), one obtains, after a few manipulations,

$$
p^{K}(\boldsymbol{x})=\sum_{i} p_{i} \varphi_{i}^{\mathscr{P} 1}(\boldsymbol{x})+\sum_{e} \frac{1}{2} \Delta\left(\boldsymbol{\Lambda}^{-1} \boldsymbol{\beta}\right)_{e} \cdot \Delta \boldsymbol{x}_{e} \varphi_{1}^{e, \mathscr{P}^{1}}(\boldsymbol{x}) \varphi_{2}^{e, \mathscr{P}^{1}}(\boldsymbol{x})
$$

where now only $\mathscr{P}^{1}$-shape functions are involved, and $\Delta t$ denotes the difference of the nodal values of a given vector $\boldsymbol{t}$ along an edge $e$. Note that the second sum over the edges of the element $K$ contains the remainder of the $\mathscr{P}^{2}$ correction associated to the product of test functions centered at each node of the edge $e$.

Remark 5. The definition (43) of the enrichment is also valid for tetrahedra.

We will now define the new pressure-enriched schemes, in which $p^{*}$ will denote the enriched pressure (43). We first propose a formulation akin the ones in $[35,36]$, in which $(13,32)$ are modified by replacing directly $p^{*}$ for the pressure in the bilinear forms. As numerically shown in Section 6, such formulations recover the desired accuracy once proper stabilization procedures are applied.

We will then elaborate on this formulation proposing a symmetric enrichment, where both the trial and test function spaces are modified. This approach yields a natural stabilization mechanism without requiring additional ad-hoc terms in the variational form, and can be elegantly proved to satisfy the desired consistency, stability, and error estimate by simple generalizations of classical arguments [30, 23]. 


\subsection{Modified schemes: Non symmetric enrichment}

\subsubsection{Conformal case}

The conformal case is straightforward, in that the enrichment (43) is used in the variational formulation (13):

$$
\begin{aligned}
B_{d}\left(\boldsymbol{w}, q ; \boldsymbol{\beta}, p^{*}\right)= & B_{d}^{G a l}\left(\boldsymbol{w}, q ; \boldsymbol{\beta}, p^{*}\right)+B^{S t a b}\left(\boldsymbol{w}, q ; \boldsymbol{\beta}, p^{*}\right)=L_{d}^{G a l}(\boldsymbol{w}, q)+L^{S t a b}(\boldsymbol{w}, q) \\
B_{d}^{G a l}\left(\boldsymbol{w}, q ; \boldsymbol{\beta}, p^{*}\right)= & \left(\boldsymbol{w}, \boldsymbol{\Lambda}^{-1} \boldsymbol{\beta}\right)_{\Omega}-\left(\boldsymbol{\nabla} \cdot \boldsymbol{w}, p^{*}\right)_{\Omega}+(q, \boldsymbol{\nabla} \cdot \boldsymbol{\beta})_{\Omega} \\
& +\left\langle\llbracket \boldsymbol{w} \rrbracket,\left\{p^{*}\right\}\right\rangle_{\epsilon_{i}}-\langle\{q\}, \llbracket \boldsymbol{\beta} \rrbracket\rangle_{\epsilon_{i}}+\left\langle\llbracket q \rrbracket, \alpha_{\beta} \llbracket p^{*} \rrbracket\right\rangle_{\epsilon_{i}}+\left\langle\llbracket \boldsymbol{w} \rrbracket, \alpha_{p} \llbracket \boldsymbol{\beta} \rrbracket\right\rangle_{\mathscr{E}_{i}} \\
& +\left\langle\boldsymbol{w} \cdot \boldsymbol{n}, p^{*}\right\rangle_{\Gamma_{N}}-\langle q, \boldsymbol{\beta} \cdot \boldsymbol{n}\rangle_{\Gamma_{N}}+\left\langle q, \alpha_{\beta} p^{*}\right\rangle_{\Gamma_{D}} \\
L_{d}^{G a l}(\boldsymbol{w}, q)= & (q, \phi)_{\Omega}-\left\langle\boldsymbol{w} \cdot \boldsymbol{n}, p_{D}\right\rangle_{\Gamma_{D}}+\left\langle q, \alpha_{\beta} p_{D}\right\rangle_{\Gamma_{D}}-\left\langle q, h_{N}\right\rangle_{\Gamma_{N}}
\end{aligned}
$$

While the test functions employed are still linear, that is $(\boldsymbol{w}, q) \in S^{1}(\Omega) \times \boldsymbol{V}^{1}(\Omega)$, the enriched pressure $p^{*}$ is defined to be a quadratic approximation of the pressure, that is $p^{*} \in S^{2}(\Omega)$. Unfortunately, as the test and trial functions spaces are different, namely $(\boldsymbol{w}, q) \in \boldsymbol{V}^{1}(\Omega) \times S^{1}(\Omega)$ and $\left(\boldsymbol{\beta}, p^{*}\right) \in \boldsymbol{V}^{1} \times S^{2}(\Omega)$, a standard coercivity argument cannot be used to derive the stability bound (15) and error estimate (16), as already seen in Section 3. We can only show that the consistency estimate is improved by the enrichment, as detailed in AppendixA.

Numerical evidence detailed here and in [31,32] shows that the non-symmetric enrichment yields a third-order pressure and a second-order flux, and allows to recover, within machine precision, quadratic polynomial solutions of the Darcy flow problem (1).

Remark 6. To achieve the levels of accuracy mentioned above, additional stabilization mechanisms are however required in some cases. For example, the "div-div" stabilization is necessary to avoid spurious modes, which may originate from the enhancing terms that replace the mid-point values. The authors of [31, 32] propose to include a condition of consistency on the tangent variation of the flux on Dirichlet boundaries, adding to the weak Galerkin formulation the additional boundary penalty term

$$
\left\langle\boldsymbol{w} \cdot \boldsymbol{\tau}_{i}, \alpha_{\tau_{i}}\left(\boldsymbol{\Lambda}^{-1} \boldsymbol{\beta} \cdot \boldsymbol{\tau}_{i}+\partial_{\tau_{i}} p_{D}\right)\right\rangle_{\Gamma_{D}}
$$

where $\tau_{i}$ denotes the tangent of the boundary in the $i$ th direction, $\partial_{\tau_{i}} p_{D}=\boldsymbol{\nabla} p_{D} \cdot \tau_{i}$ is the gradient of the Dirichlet value along the boundary, and the penalty coefficient $\alpha_{\tau}$ is defined as $\alpha_{\tau}=\tilde{\alpha} h /\|\boldsymbol{\Lambda}\|$. Such penalty term has a behavior similar to the "div-div" stabilization term $\frac{\zeta}{2}\left(\boldsymbol{\nabla} \cdot \boldsymbol{w},\|\boldsymbol{\Lambda}\| h^{2} \boldsymbol{\nabla} \cdot \boldsymbol{\beta}\right)_{\Omega}$ in $B^{S t a b}(\boldsymbol{w}, q ; \boldsymbol{\beta}, p)$. For this reason, the "div-div" stabilization can be removed if (45) is employed.

\subsubsection{Embedded case: third-order extrapolation}

In the embedded case, the boundary terms of the type $\left\langle q, \alpha_{\beta}\left(p-p_{D}\right)\right\rangle_{\Gamma_{D}}+\left\langle\boldsymbol{w} \cdot \boldsymbol{n}, p_{D}\right\rangle_{\Gamma_{D}}$ need to be modified to account for a parabolic extrapolation, in order to be consistent with the enrichment of the pressure. For this reason, when extending the value of the pressure from $\Gamma_{D}$ to $\tilde{\Gamma}_{D}$, the Taylor expansion (30) has been enhanced using the Hessian of the pressure:

$$
p_{D}(\boldsymbol{x})=\bar{p}_{D}=p_{D}(\tilde{\boldsymbol{x}})+\nabla p \cdot \boldsymbol{d}+\frac{1}{2} \boldsymbol{d}^{T} \mathscr{H}(p) \boldsymbol{d}
$$

As in the case of internal edges, to evaluate the Hessian we use the nodal flux, which essentially leads to (39) with $\boldsymbol{\delta} \boldsymbol{x}=\boldsymbol{d}$. The resulting shifted boundary condition reads:

$$
p_{D}(\boldsymbol{x})=\bar{p}_{D}=p_{D}(\tilde{\boldsymbol{x}})-\left(\boldsymbol{\Lambda}^{-1} \boldsymbol{\beta}\right) \cdot \boldsymbol{d}-\frac{1}{2} \boldsymbol{d}^{T} \boldsymbol{\nabla}\left(\boldsymbol{\Lambda}^{-1} \boldsymbol{\beta}\right) \boldsymbol{d}
$$

Thus, the Dirichlet terms are now modified as follows:

$$
\left\langle q-\boldsymbol{\Lambda}^{-1} \boldsymbol{w} \cdot \boldsymbol{d}-\frac{1}{2} \boldsymbol{d}^{T} \boldsymbol{\nabla}\left(\boldsymbol{\Lambda}^{-1} \boldsymbol{w}\right) \boldsymbol{d}, \alpha_{\beta}\left(p-\boldsymbol{\Lambda}^{-1} \boldsymbol{\beta} \cdot \boldsymbol{d}-\frac{1}{2} \boldsymbol{d}^{T} \boldsymbol{\nabla}\left(\boldsymbol{\Lambda}^{-1} \boldsymbol{\beta}\right) \boldsymbol{d}-\bar{p}_{D}\right)\right\rangle_{\tilde{\Gamma}_{D}}+\left\langle\boldsymbol{w} \cdot \tilde{\boldsymbol{n}}, \boldsymbol{\Lambda}^{-1} \boldsymbol{\beta} \cdot \boldsymbol{d}+\frac{1}{2} \boldsymbol{d}^{T} \boldsymbol{\nabla}\left(\boldsymbol{\Lambda}^{-1} \boldsymbol{\beta}\right) \boldsymbol{d}+\bar{p}_{D}\right\rangle_{\tilde{\Gamma}_{D}}
$$

where the expression in the left slot of the first term is designed to guarantee the symmetry and positive semidefiniteness of the associated variational form, in the spirit of the formulation used in [28]. 
Since no modification is performed on the flux itself, the Neumann boundary terms remain unchanged, and the final embedded formulation using pressure enrichment reads:

$$
\begin{aligned}
B_{d}\left(\boldsymbol{w}, q ; \boldsymbol{\beta}, p^{*}\right)= & B_{d}^{G a l}\left(\boldsymbol{w}, q ; \boldsymbol{\beta}, p^{*}\right)+B^{S t a b}\left(\boldsymbol{w}, q ; \boldsymbol{\beta}, p^{*}\right)=L_{d}^{G a l}(\boldsymbol{w}, q)+L^{S t a b}(\boldsymbol{w}, q) \\
B_{d}^{G a l}\left(\boldsymbol{w}, q ; \boldsymbol{\beta}, p^{*}\right)= & \left(\boldsymbol{w}, \boldsymbol{\Lambda}^{-1} \boldsymbol{\beta}\right)_{\tilde{\Omega}}-\left(\boldsymbol{\nabla} \cdot \boldsymbol{w}, p^{*}\right)_{\tilde{\Omega}}+(q, \boldsymbol{\nabla} \cdot \boldsymbol{\beta})_{\tilde{\Omega}} \\
& +\left\langle\llbracket \boldsymbol{w} \rrbracket,\left\{p^{*}\right\}\right\rangle_{\epsilon_{i}}-\langle\{q\}, \llbracket \boldsymbol{\beta} \rrbracket\rangle_{\epsilon_{i}}+\left\langle\llbracket q \rrbracket, \alpha_{\beta} \llbracket p^{*} \rrbracket\right\rangle_{\epsilon_{i}}+\left\langle\llbracket \boldsymbol{w} \rrbracket, \alpha_{p} \llbracket \boldsymbol{\beta} \rrbracket\right\rangle_{\mathscr{E}_{i}} \\
& +\left\langle q-\boldsymbol{\Lambda}^{-1} \boldsymbol{w} \cdot \boldsymbol{d}-\frac{1}{2} \boldsymbol{d}^{T} \boldsymbol{\nabla}\left(\boldsymbol{\Lambda}^{-1} \boldsymbol{w}\right) \boldsymbol{d}, \alpha_{\beta}\left(p-\boldsymbol{\Lambda}^{-1} \boldsymbol{\beta} \cdot \boldsymbol{d}-\frac{1}{2} \boldsymbol{d}^{T} \boldsymbol{\nabla}\left(\boldsymbol{\Lambda}^{-1} \boldsymbol{\beta}\right) \boldsymbol{d}\right)\right\rangle_{\tilde{\Gamma}_{D}} \\
& +\left\langle\boldsymbol{w} \cdot \tilde{\boldsymbol{n}}, \boldsymbol{\Lambda}^{-1} \boldsymbol{\beta} \cdot \boldsymbol{d}+\frac{1}{2} \boldsymbol{d}^{T} \boldsymbol{\nabla}\left(\boldsymbol{\Lambda}^{-1} \boldsymbol{\beta}\right) \boldsymbol{d}\right\rangle_{\tilde{\Gamma}_{D}}+\left\langle\boldsymbol{w} \cdot \tilde{\boldsymbol{n}}, p^{*}\right\rangle_{\tilde{\Gamma}_{N}}-\langle q(\boldsymbol{n} \cdot \tilde{\boldsymbol{n}}),(\boldsymbol{\beta}+[\boldsymbol{\nabla} \boldsymbol{\beta}] \boldsymbol{d}) \cdot \boldsymbol{n}\rangle_{\tilde{\Gamma}_{N}} \\
L_{d}^{G a l}(\boldsymbol{w}, q)= & (q, \phi)_{\tilde{\Omega}}-\left\langle q(\boldsymbol{n} \cdot \tilde{\boldsymbol{n}}), \bar{h}_{N}\right\rangle_{\tilde{\Gamma}_{N}}-\left\langle\boldsymbol{w} \cdot \tilde{\boldsymbol{n}}, \bar{p}_{D}\right\rangle_{\tilde{\Gamma}_{D}}+\left\langle q-\boldsymbol{\Lambda}^{-1} \boldsymbol{w} \cdot \boldsymbol{d}-\frac{1}{2} \boldsymbol{d}^{T} \boldsymbol{\nabla}\left(\boldsymbol{\Lambda}^{-1} \boldsymbol{w}\right) \boldsymbol{d}, \alpha_{\beta} \bar{p}_{D}\right\rangle_{\tilde{\Gamma}_{D}}
\end{aligned}
$$

As before, the continuous Galerkin formulation is obtained by removing all internal edge integrals involving jumps and is omitted for the sake brevity. The Euler-Lagrange equations associated to the enriched scheme read:

$$
\begin{aligned}
0= & \left(\boldsymbol{w},\left(\boldsymbol{\Lambda}^{-1} \boldsymbol{\beta}+\boldsymbol{\nabla} p^{*}\right)\right)_{\tilde{\Omega}}+(q,(\boldsymbol{\nabla} \cdot \boldsymbol{\beta}-\phi))_{\tilde{\Omega}} \\
& -\left\langle\boldsymbol{w} \cdot \tilde{\boldsymbol{n}}+\alpha_{\beta}\left(q-\boldsymbol{\Lambda}^{-1} \boldsymbol{w} \cdot \boldsymbol{d}-\frac{1}{2} \boldsymbol{d}^{T} \boldsymbol{\nabla}\left(\boldsymbol{\Lambda}^{-1} \boldsymbol{w}\right) \boldsymbol{d}\right), p^{*}-\boldsymbol{\Lambda}^{-1} \boldsymbol{\beta} \cdot \boldsymbol{d}-\frac{1}{2} \boldsymbol{d}^{T} \boldsymbol{\nabla}\left(\boldsymbol{\Lambda}^{-1} \boldsymbol{\beta}\right) \boldsymbol{d}-\bar{p}_{D}\right\rangle_{\tilde{\Gamma}_{D}} \\
& -\left\langle q(\boldsymbol{n} \cdot \tilde{\boldsymbol{n}}),(\boldsymbol{\beta}+(\boldsymbol{\nabla} \boldsymbol{\beta}) \boldsymbol{d}) \cdot \boldsymbol{n}-\bar{h}_{N}\right\rangle_{\tilde{\Gamma}_{N}}
\end{aligned}
$$

This Euler-Lagrange formulation differs from (34), due to the use of the enriched pressure and the mixed formulation to evaluate pressure gradients in the boundary terms. On $\tilde{\Gamma}_{D}$, to enforce with higher accuracy the boundary condition, the Taylor expansion is enhanced to third order. As illustrated by the numerical examples of Section 6, such a scheme allows to recover overall second-order accuracy.

Remark 7. The extrapolation of the pressure could be already enhanced to third order without the pressure enrichment. However, such a procedure is unnecessary as the expected accuracy is second order and is already satisfied by a second-order Taylor expansion.

Remark 8. When employed, the additional high-order correction on the tangential gradient (45) is also extended using the Taylor expansion:

$$
\partial_{\tau_{i}} \bar{p}_{D}=\boldsymbol{\nabla} \bar{p}_{D} \cdot \tau_{i}=\boldsymbol{\Lambda}^{-1} \boldsymbol{\beta}(\tilde{\boldsymbol{x}}) \cdot \boldsymbol{\tau}_{i}+\boldsymbol{\nabla}\left(\boldsymbol{\Lambda}^{-1} \boldsymbol{\beta}\right) \boldsymbol{d} \cdot \boldsymbol{\tau}_{i}+\mathscr{O}\left(\|\boldsymbol{d}\|^{2}\right)
$$

\subsection{Modification of the schemes - Symmetric enrichment}

We propose next an improved variant of the ideas discussed so far. For this purpose, let us introduce the mapping $\mathscr{R}_{l}^{l+1}:\left(\mathbf{V}^{k}(\Omega), S^{l}(\Omega)\right) \mapsto S^{l+1}(\Omega)$, which defines the enriched pressure in the case of linear approximations, $(\boldsymbol{\beta}, p) \in$ $\mathbf{V}^{1}(\Omega) \times S^{1}(\Omega)$ :

$$
\begin{aligned}
\mathscr{R}_{1}^{2}(\Omega):\left(\mathbf{V}^{1}(\Omega), S^{1}(\Omega)\right) & \mapsto S^{2}(\Omega) \\
(\boldsymbol{\beta}, p) & \rightarrow p *
\end{aligned}
$$

\subsubsection{Conformal case}

We propose here a symmetrically enriched method in which the operator (52) is used on both the primary variable (the pressure) and on the corresponding test function $q$ in the bilinear form:

$$
B_{d}\left(\boldsymbol{w}, \mathscr{R}_{1}^{2}(\boldsymbol{w}, q) ; \boldsymbol{\beta}, \mathscr{R}_{1}^{2}(\boldsymbol{\beta}, q)\right)=L_{d}\left(\boldsymbol{w}, \mathscr{R}_{1}^{2}(\boldsymbol{w}, q)\right)
$$


With respect to the previous non-symmetric enriched formulation, now the test and trial spaces are identical. Denoting $q^{*}=\mathscr{R}_{1}^{2}(\boldsymbol{w}, q)$, we consider the following stabilized variational form:

$$
\begin{aligned}
B_{d}\left(\boldsymbol{w}, q^{*} ; \boldsymbol{\beta}, p^{*}\right)= & B_{d}^{G a l}\left(\boldsymbol{w}, q^{*} ; \boldsymbol{\beta}, p^{*}\right)+B^{S t a b}\left(\boldsymbol{w}, q^{*} ; \boldsymbol{\beta}, p^{*}\right)=L_{d}^{G a l}\left(\boldsymbol{w}, q^{*}\right) \\
B_{d}^{G a l}\left(\boldsymbol{w}, q^{*} ; \boldsymbol{\beta}, p^{*}\right)= & \left(\boldsymbol{w}, \boldsymbol{\Lambda}^{-1} \boldsymbol{\beta}\right)_{\Omega}-\left(\boldsymbol{\nabla} \cdot \boldsymbol{w}, p^{*}\right)_{\Omega}+\left(q^{*}, \boldsymbol{\nabla} \cdot \boldsymbol{\beta}\right)_{\Omega} \\
& +\left\langle\llbracket \boldsymbol{w} \rrbracket,\left\{p^{*}\right\}\right\rangle_{\epsilon_{i}}-\left\langle\left\{q^{*}\right\}, \llbracket \boldsymbol{\beta} \rrbracket\right\rangle_{\epsilon_{i}}+\left\langle\llbracket q^{*} \rrbracket, \alpha_{\beta} \llbracket p^{*} \rrbracket\right\rangle_{\epsilon_{i}}+\left\langle\llbracket \boldsymbol{w} \rrbracket, \alpha_{p} \llbracket \boldsymbol{\beta} \rrbracket\right\rangle_{\mathcal{E}_{i}} \\
& +\left\langle\boldsymbol{w} \cdot \boldsymbol{n}, p^{*}\right\rangle_{\Gamma_{N}}-\left\langle q^{*}, \boldsymbol{\beta} \cdot \boldsymbol{n}\right\rangle_{\Gamma_{N}}+\left\langle q^{*}, \alpha_{\beta} p^{*}\right\rangle_{\Gamma_{D}} \\
B^{S t a b}\left(\boldsymbol{w}, q^{*}, \boldsymbol{\beta}, p^{*}\right)= & \frac{1}{2}\left(-\boldsymbol{\Lambda}^{-1} \boldsymbol{w}+\boldsymbol{\nabla} q^{*}, \boldsymbol{\beta}+\boldsymbol{\Lambda} \boldsymbol{\nabla} p^{*}\right)_{\Omega} \\
L_{d}^{G a l}\left(\boldsymbol{w}, q^{*}\right)= & \left(q^{*}, \phi\right)_{\Omega}-\left\langle\boldsymbol{w} \cdot \boldsymbol{n}, p_{D}\right\rangle_{\Gamma_{D}}+\left\langle q^{*}, \alpha_{\beta} p_{D}\right\rangle_{\Gamma_{D}}-\left\langle q^{*}, h_{N}\right\rangle_{\Gamma_{N}}
\end{aligned}
$$

The proposed symmetric enriched formulation allows for a more straightforward analysis. Setting $\boldsymbol{W}=(\boldsymbol{w}, q), \boldsymbol{W}^{*}=$ $\left(\boldsymbol{w}, \mathscr{R}_{1}^{2}(\boldsymbol{w}, q)\right)$ and indicating by $\boldsymbol{V}_{\boldsymbol{e}}=\left(\boldsymbol{\beta}_{e}, p_{e}\right)$ a sufficiently smooth solution, the following theorem provides stability, consistency and accuracy of the formulation (for the proof, see AppendixA):

Theorem 1 (Symmetric enrichment - stability, consistency and error estimate). For all $\left(\boldsymbol{w}, q^{*}\right)$ and $\left(\boldsymbol{\beta}, p^{*}\right) \in \boldsymbol{V}_{1} \times$ $\mathscr{R}_{1}^{2}\left(V_{1}, S_{1}(\Omega)\right)$, the enriched formulation (54) is:

a) stable (coercive) with respect to the norm

$$
\begin{aligned}
\left\|\left(\boldsymbol{\beta}, p^{*}\right)\right\| \|^{2}= & \frac{1}{2}\left(\left\|\boldsymbol{\Lambda}^{-\mathbf{1} / \mathbf{2}} \boldsymbol{\beta}\right\|_{\Omega}^{2}+\left\|\boldsymbol{\Lambda}^{\mathbf{1 / 2}} \nabla p^{*}\right\|_{\Omega}^{2}\right) \\
& +\left\|\left(\tilde{\alpha}_{\beta}\|\mathbf{\Lambda}\| / h^{\perp}\right)^{1 / 2} \llbracket p^{*} \rrbracket\right\|_{\mathscr{E}_{i}}^{2}+\left\|\left(\tilde{\alpha}_{\beta}\|\boldsymbol{\Lambda}\| / h^{\perp}\right)^{1 / 2} p^{*}\right\|_{\Gamma_{D}}^{2}+\left\|\left(\tilde{\alpha}_{p} h^{\perp} /\|\boldsymbol{\Lambda}\|\right)^{1 / 2} \llbracket \boldsymbol{\beta} \rrbracket\right\|^{2}
\end{aligned}
$$

b) consistent (in the sense of the Galerkin orthogonality property), that is

$$
B_{d}\left(\boldsymbol{W}^{*}, \boldsymbol{E}\right)=0
$$

c) convergent, with the error satisfying the estimate

$$
\|\left.|\boldsymbol{E}|\left|\leq C_{1} h^{2}\right| \beta\right|_{2}+C_{2} h^{2}|p|_{3}
$$

where $\boldsymbol{E}=\left(\boldsymbol{\beta}-\boldsymbol{\beta}_{e} ; p^{*}-p_{e}\right)$.

This last error estimates differs from (16), since an extra order of accuracy is gained for the flux, namely $r=2$. One additional order of convergence is also expected for the $L^{2}$-error of the pressure. In conclusion the expected rates of convergence for pressure and flux are third and second order, respectively.

Remark 9. In the discontinuous case, the $B^{S t a b}\left(\boldsymbol{w}, q^{*}, \boldsymbol{\beta}, p^{*}\right)$ term is not necessary. For a continuous approximation, the "div-div" term was found necessary for the non-symmetric scheme, but unnecessary for the symmetric scheme, for which the momentum stabilization is sufficient to guarantee stability and convergence, as also confirmed in a wide variety of computations. In fact, the symmetric enrichment naturally introduces a stabilization mechanism akin to the "div-div" term:

$$
\begin{aligned}
\frac{1}{2}\left(-\boldsymbol{\Lambda}^{-1} \boldsymbol{w}+\boldsymbol{\nabla} q^{*}, \boldsymbol{\beta}+\boldsymbol{\Lambda} \boldsymbol{\nabla} p^{*}\right)_{\Omega}= & \frac{1}{2}\left(-\boldsymbol{\Lambda}^{-1} \boldsymbol{w}+\boldsymbol{\nabla} q, \boldsymbol{\beta}+\boldsymbol{\Lambda} \boldsymbol{\nabla} p^{*}\right)_{\Omega}+\frac{1}{2}\left(\boldsymbol{\nabla}\left(q^{*}-q\right), \boldsymbol{\beta}+\boldsymbol{\Lambda} \boldsymbol{\nabla} p\right)_{\Omega} \\
& +\frac{1}{2}\left(\boldsymbol{\nabla}\left(q^{*}-q\right), \boldsymbol{\beta}+\boldsymbol{\Lambda} \boldsymbol{\nabla}\left(p^{*}-p\right)\right)_{\Omega}
\end{aligned}
$$

Focusing on the last term and using (43),

$$
\begin{aligned}
\frac{1}{2}\left(\boldsymbol{\nabla}\left(q^{*}-q\right), \boldsymbol{\beta}+\boldsymbol{\Lambda} \boldsymbol{\nabla}\left(p^{*}-p\right)\right)_{\Omega} & =\sum_{K \in \Omega} \frac{1}{2}\left(\boldsymbol{\nabla}\left(q^{*}-q\right), \boldsymbol{\beta}+\boldsymbol{\Lambda} \boldsymbol{\nabla}\left(p^{*}-p\right)\right)_{K} \\
& =\sum_{K \in \Omega} \frac{1}{8}\left(\sum_{e} \boldsymbol{\nabla}\left(\Delta\left(\boldsymbol{\Lambda}^{-\mathbf{1}} \boldsymbol{w}\right)_{e} \cdot \Delta \boldsymbol{x}_{e} \varphi_{1}^{e} \varphi_{2}^{e}\right), \boldsymbol{\Lambda} \sum_{e} \boldsymbol{\nabla}\left(\Delta\left(\boldsymbol{\Lambda}^{-\mathbf{1}} \boldsymbol{\beta}\right)_{e} \cdot \Delta \boldsymbol{x}_{e} \varphi_{1}^{e} \varphi_{2}^{e}\right)\right)_{K}
\end{aligned}
$$


This last term in equality (59) contains the inner product of the gradient of the flux and the gradient of the shape function, similar in nature to the "div-div" stabilization. Note also that the scaling with the square of the grid size $\left(h^{2}\right)$ is accounted for through the dot products with $\Delta \boldsymbol{x}_{e}$. Thus, when mapping from the linear unknowns to the quadratic pressure, the "div-div" stabilization is directly included in the momentum stabilization and can be removed from the variational form. This provides a natural and order-preserving stabilization, independent from any stabilization constant.

\subsubsection{Embedded case}

Proceeding as above, an enhanced extrapolation is applied to the SBM in order to preserve the required order of accuracy. This leads to the following stabilized variational form:

$$
\begin{aligned}
B_{d}\left(\boldsymbol{w}, q^{*} ; \boldsymbol{\beta}, p^{*}\right)= & B_{d}^{G a l}\left(\boldsymbol{w}, q^{*} ; \boldsymbol{\beta}, p^{*}\right)+B^{S t a b}\left(\boldsymbol{w}, q^{*} ; \boldsymbol{\beta}, p^{*}\right)=L_{d}^{G a l}\left(\boldsymbol{w}, q^{*}\right)+L^{S t a b}\left(\boldsymbol{w}, q^{*}\right) \\
B_{d}^{G a l}\left(\boldsymbol{w}, q^{*} ; \boldsymbol{\beta}, p^{*}\right)= & \left(\boldsymbol{w}, \boldsymbol{\Lambda}^{-1} \boldsymbol{\beta}\right)_{\tilde{\Omega}}-\left(\boldsymbol{\nabla} \cdot \boldsymbol{w}, p^{*}\right)_{\tilde{\Omega}}+\left(q^{*}, \boldsymbol{\nabla} \cdot \boldsymbol{\beta}\right)_{\tilde{\Omega}} \\
& +\left\langle\llbracket \boldsymbol{w} \rrbracket,\left\{p^{*}\right\}\right\rangle_{\epsilon_{i}}-\left\langle\left\{q^{*}\right\}, \llbracket \boldsymbol{\beta} \rrbracket\right\rangle_{\epsilon_{i}}+\left\langle\llbracket q^{*} \rrbracket, \alpha_{\beta} \llbracket p^{*} \rrbracket\right\rangle_{\epsilon_{i}}+\left\langle\llbracket \boldsymbol{w} \rrbracket, \alpha_{p} \llbracket \boldsymbol{\beta} \rrbracket\right\rangle_{\tilde{E}_{i}} \\
& +\left\langle q^{*}-\boldsymbol{\Lambda}^{-1} \boldsymbol{w} \cdot \boldsymbol{d}-\frac{1}{2} \boldsymbol{d}^{T} \boldsymbol{\nabla}\left(\boldsymbol{\Lambda}^{-1} \boldsymbol{w}\right) \boldsymbol{d}, \alpha_{\beta}\left(p-\boldsymbol{\Lambda}^{-1} \boldsymbol{\beta} \cdot \boldsymbol{d}-\frac{1}{2} \boldsymbol{d}^{T} \boldsymbol{\nabla}\left(\boldsymbol{\Lambda}^{-1} \boldsymbol{\beta}\right) \boldsymbol{d}\right)_{\tilde{\Gamma}_{D}}\right. \\
& +\left\langle\boldsymbol{w} \cdot \tilde{\boldsymbol{n}}, \boldsymbol{\Lambda}^{-1} \boldsymbol{\beta} \cdot \boldsymbol{d}+\frac{1}{2} \boldsymbol{d}^{T} \boldsymbol{\nabla}\left(\boldsymbol{\Lambda}^{-1} \boldsymbol{\beta}\right) \boldsymbol{d}\right\rangle_{\tilde{\Gamma}_{D}}+\left\langle\boldsymbol{w} \cdot \tilde{\boldsymbol{n}}, p^{*}\right\rangle_{\tilde{\Gamma}_{N}}-\left\langle q^{*}(\boldsymbol{n} \cdot \tilde{\boldsymbol{n}}),(\boldsymbol{\beta}+[\boldsymbol{\nabla} \boldsymbol{\beta}] \boldsymbol{d}) \cdot \boldsymbol{n}\right\rangle_{\tilde{\Gamma}_{N}} \\
L_{d}^{G a l}\left(\boldsymbol{w}, q^{*}\right)= & \left(q^{*}, \phi\right)_{\tilde{\Omega}}-\left\langle q^{*}(\boldsymbol{n} \cdot \tilde{\boldsymbol{n}}), \bar{h}_{N}\right\rangle_{\tilde{\Gamma}_{N}}-\left\langle\boldsymbol{w} \cdot \tilde{\boldsymbol{n}}, \bar{p}_{D}\right\rangle_{\tilde{\Gamma}_{D}}+\left\langle q^{*}-\boldsymbol{\Lambda}^{-1} \boldsymbol{w} \cdot \boldsymbol{d}-\frac{1}{2} \boldsymbol{d}^{T} \boldsymbol{\nabla}\left(\boldsymbol{\Lambda}^{-1} \boldsymbol{w}\right) \boldsymbol{d}, \alpha_{\beta} \bar{p}_{D}\right\rangle_{\tilde{\Gamma}_{D}}
\end{aligned}
$$

Here, as for the original SBM formulation (32) and for the non-symmetric formulation (49), we do expect a reduction in accuracy for the pressure when Neumann conditions are embedded. However, the overall accuracy is still secondorder for both flux and pressure. Hence, this enriched scheme and its non-symmetric counterpart deliver second-order accuracy for the flux and pressure, for any kind of embedded boundary conditions. In addition, if only Dirichlet conditions are embedded, the approximated pressure is third-order accurate.

\section{Numerical results}

In this section, several numerical examples are presented, to confirm the the theoretical expectations, as well as to provide further insights on the previous discussion on the accuracy and stability of the schemes.

For all two- and three-dimensional simulations, the results are obtained with the symmetric enriched method, using, where necessary, penalty coefficients set to $\alpha_{p}=2$ and $\alpha_{\beta}=1$. The non-symmetric formulation is only used in the convergence tests and in comparative studies among the different options for stabilization.

\subsection{Convergence tests}

The convergence tests presented next aim at demonstrating the accuracy of the proposed schemes, and, in the case of the pressure enrichment, tradeoffs between accuracy and overall computational cost. In addition, for Dirichlet boundary conditions, we also illustrate the differences between the formulations and, for the non-symmetric enrichment, the importance of the "div-div" stabilization or the tangential penalty term.

\subsubsection{Setup of the tests cases}

We use the method of manufactured solutions with matching boundary conditions on a geometry given by the circular annulus of outer radius $r_{\text {out }}=0.35$ and inner radius $r_{\text {in }}=0.1$ (see Fig. 3(a)). Note that in one of the tests for the embedded case, the inner circular boundary shape is replaced by a square shape of size $l=0.15$ (see Fig. 3(c)), with the goal of demonstrating the robustness of the approach on geometries with singularities. The manufactured 


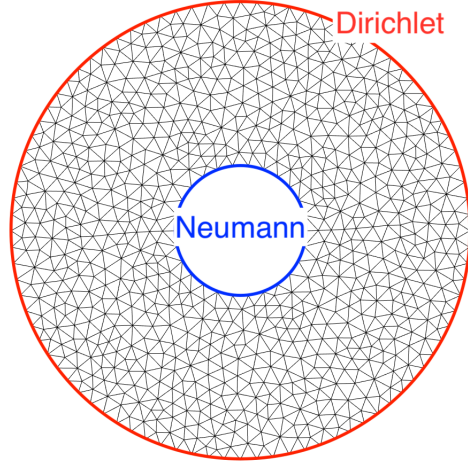

(a) Conformal simulation domain and mesh

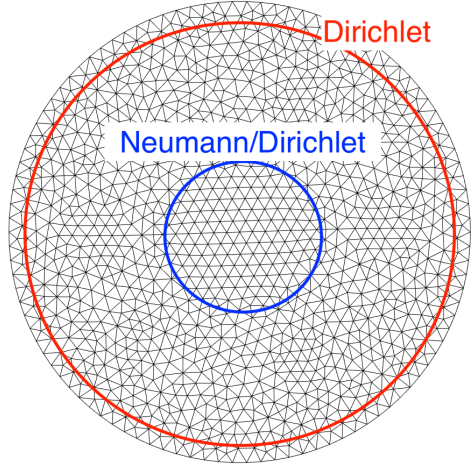

(b) Embedded simulation with inner embbeded circle, domain and mesh

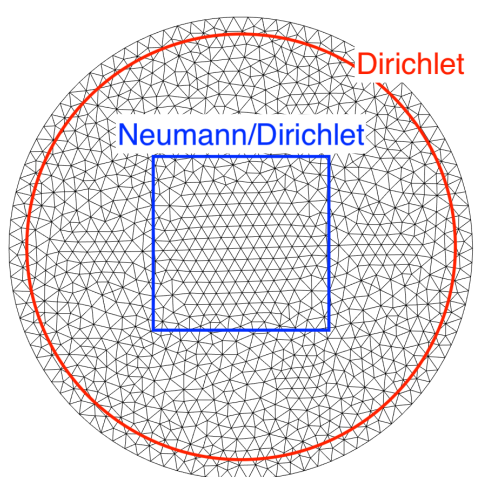

(c) Embedded simulation with inner embbeded square, domain and mesh

Figure 3: Typical grids and geometries for the convergence tests.

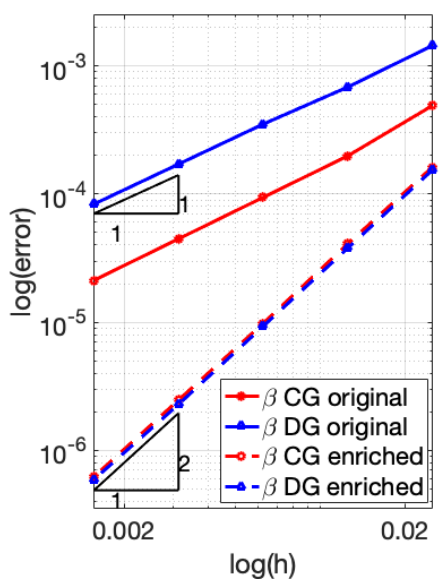

(a) Flux - Isotropic permeability

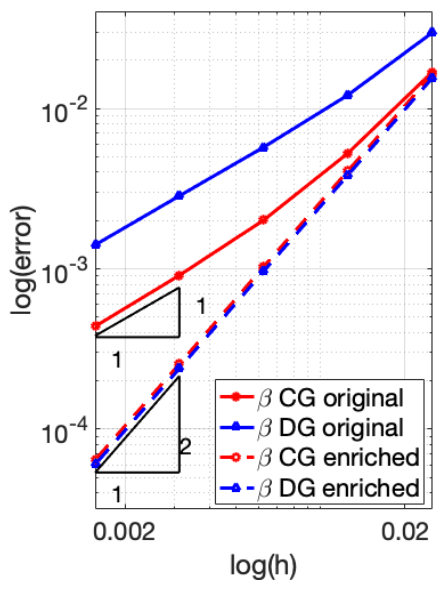

(c) Flux - Anisotropic permeability

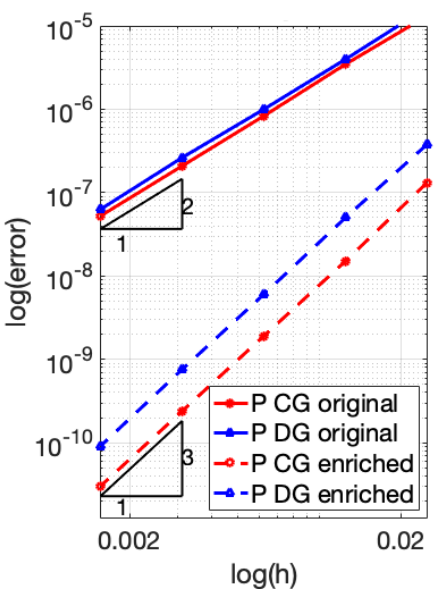

(b) Pressure - Isotropic permeability

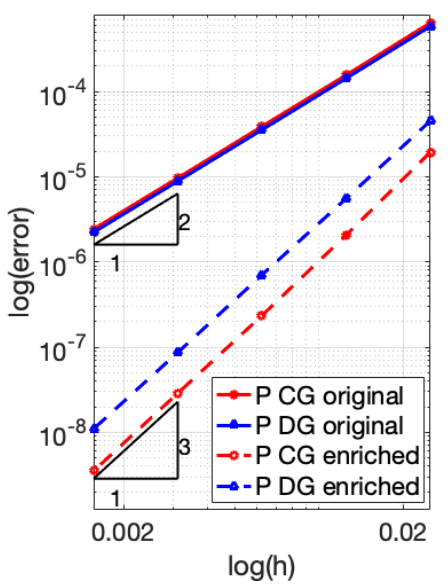

(d) Pressure - Anisotropic permeability

Figure 4: Convergence test: Conformal case. 


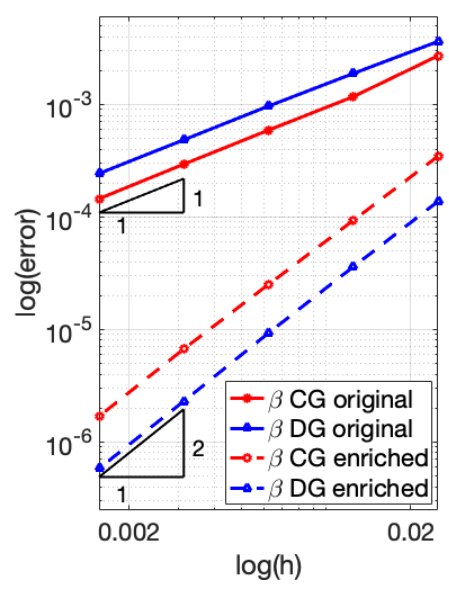

(a) Flux: Isotropic permeability $\boldsymbol{\Lambda}$

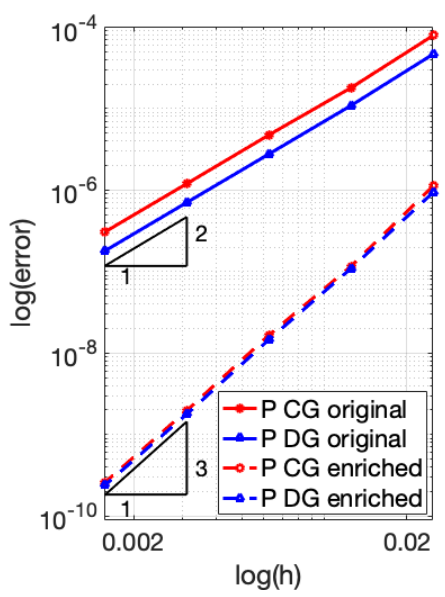

(d) Pressure: Isotropic permeability $\boldsymbol{\Lambda}$

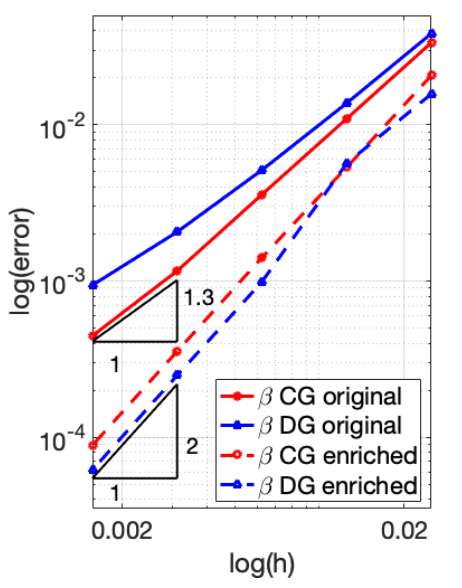

(b) Flux: Anisotropic permeability $\boldsymbol{\Lambda}$ - Circular inner boundary

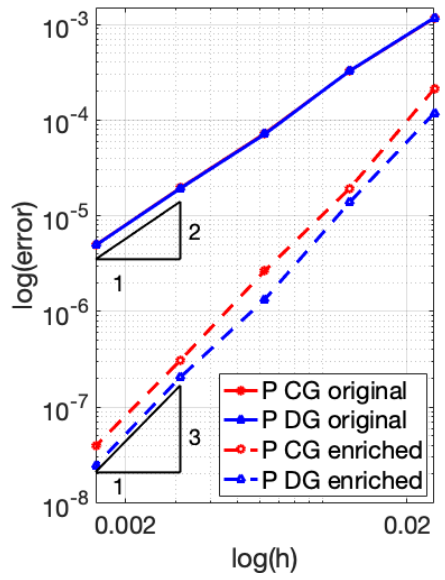

(e) Pressure: Anisotropic permeability $\mathbf{\Lambda}$ Circular inner boundary

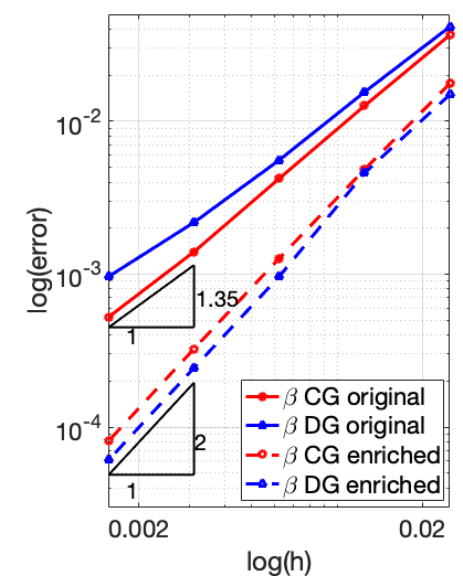

(c) Flux: Anisotropic permeability $\boldsymbol{\Lambda}$ Square inner boundary

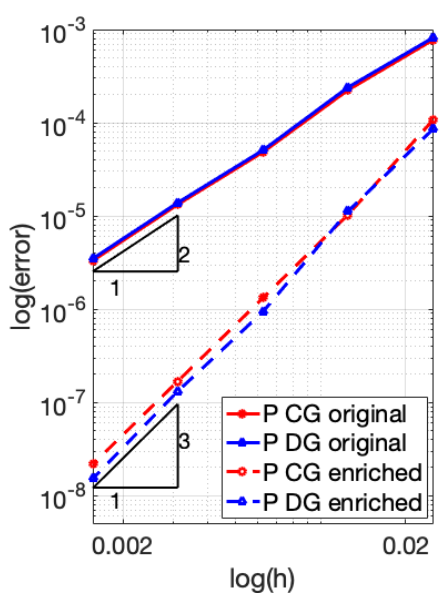

(f) Pressure: Anisotropic permeability $\boldsymbol{\Lambda}$ Square inner boundary

Figure 5: Convergence test for the embedded simulations: Dirichlet/Dirichlet.

solutions employed for this study are defined as

$$
\left\{\begin{aligned}
\boldsymbol{\beta}(x, y) & =\left(\begin{array}{c}
-e^{x+y}\left(x^{2}+y\right) \\
-e^{x+y}\left(x+y^{2}\right)
\end{array}\right) \\
p(x, y) & =\frac{1}{3}\left(x^{3}+y^{3}\right)+x y \\
\phi(x, y) & =-\left(y^{2}+x^{2}+3 x+3 y\right) e^{x+y} \\
\boldsymbol{\Lambda} & =e^{x+y} \mathbb{I}
\end{aligned}\right.
$$

for the variable permeability case, and as 


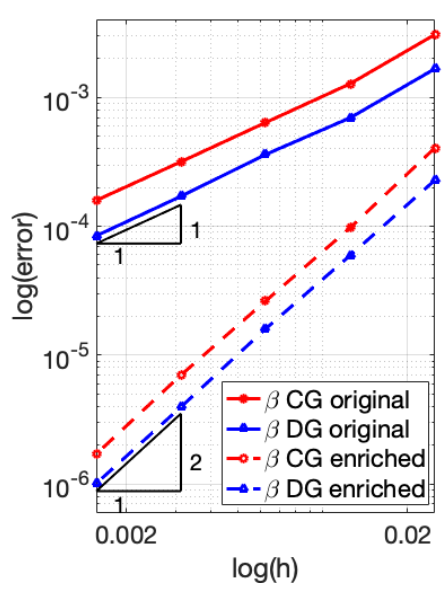

(a) Flux: Isotropic permeability $\boldsymbol{\Lambda}$

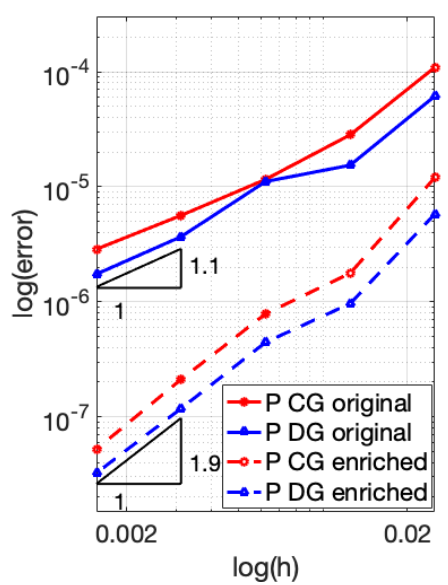

(d) Pressure: Isotropic permeability $\boldsymbol{\Lambda}$

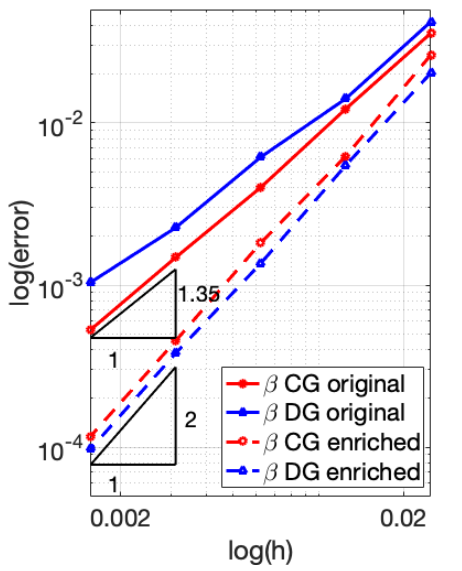

(b) Flux: Anisotropic permeability $\boldsymbol{\Lambda}$ - Circular inner boundary

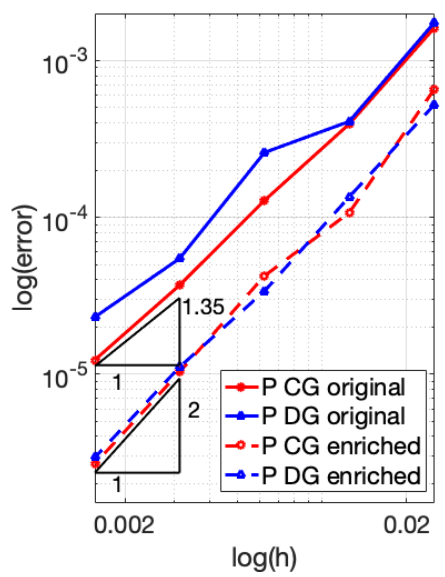

(e) Pressure: Anisotropic permeability $\boldsymbol{\Lambda}$ Circular inner boundary

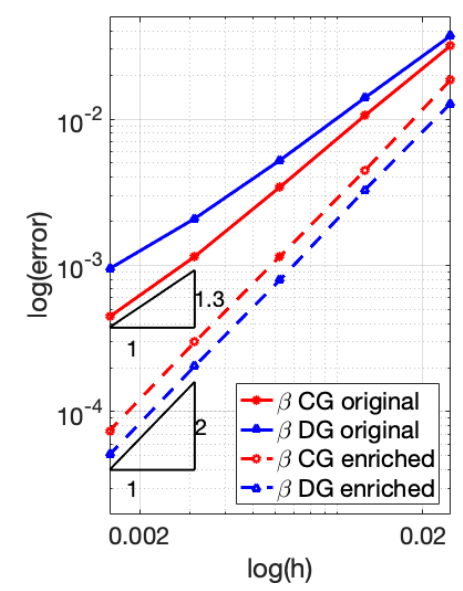

(c) Flux: Anisotropic permeability $\boldsymbol{\Lambda}$ Square inner boundary

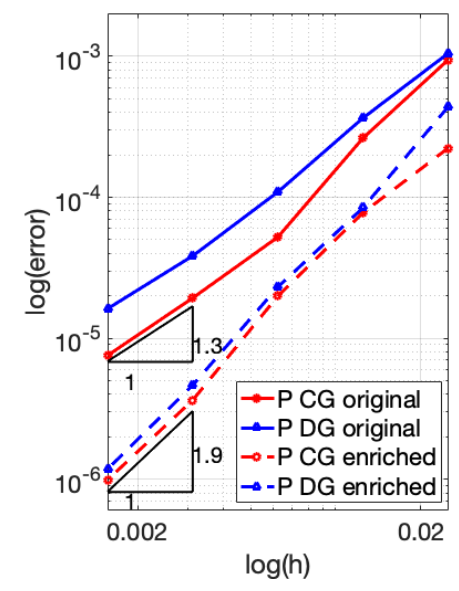

(f) Pressure: Anisotropic permeability $\boldsymbol{\Lambda}$ Square inner boundary

Figure 6: Convergence test for the embedded simulations: Neumann/Dirichlet.

$$
\left\{\begin{aligned}
\boldsymbol{\beta}(x, y) & =-\pi\left(\begin{array}{c}
\lambda_{x y} \sin (4 \pi x) \cos (4 \pi y)+\lambda_{x x} \cos (4 \pi x) \sin (4 \pi y) \\
\lambda_{y y} \sin (4 \pi x) \cos (4 \pi y)+\lambda_{y x} \cos (4 \pi x) \sin (4 \pi y)
\end{array}\right) \\
p(x, y) & =\frac{\sin (4 \pi x) \sin (4 \pi y)}{4} \\
\phi(x, y) & =4 \pi^{2}\left(\left(\lambda_{y y}+\lambda_{x x}\right) \sin (4 \pi x) \sin (4 \pi y)-\left(\lambda_{y x}+\lambda_{x y}\right) \cos (4 \pi x) \cos (4 \pi y)\right) \\
\boldsymbol{\Lambda} & =\left[\begin{array}{ll}
\lambda_{x x} & \lambda_{x y} \\
\lambda_{y x} & \lambda_{y y}
\end{array}\right]=\left[\begin{array}{ll}
3 & 1 \\
1 & 3
\end{array}\right]
\end{aligned}\right.
$$

for the anisotropic permeability case.

\subsubsection{Conformal Results}

Dirichlet and Neumann boundary conditions are applied in the outer and inner boundaries, respectively (see Fig. 3(a)). For the variable permeability case (61), convergence rates for both continuous and discontinuous discretizations are given in Figure 4(a) for the flux and Figure 4(b) for the pressure. For the anisotropic permeability case (62), 


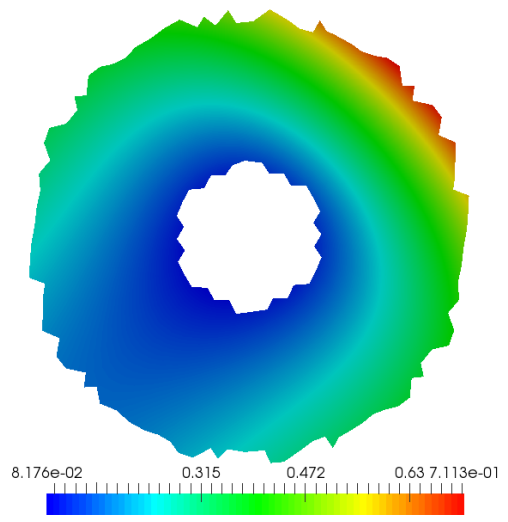

(a) Flux norm

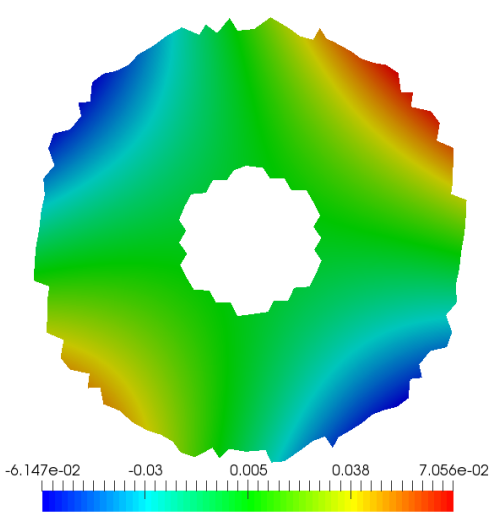

(b) Pressure

Figure 7: Convergence test: Plots of the solution for the isotropic permeability distribution.

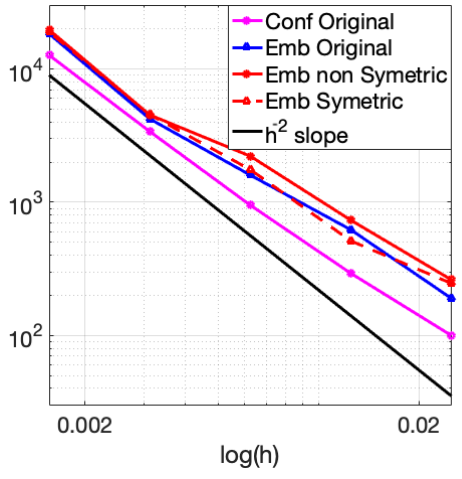

(a) Continuous scheme

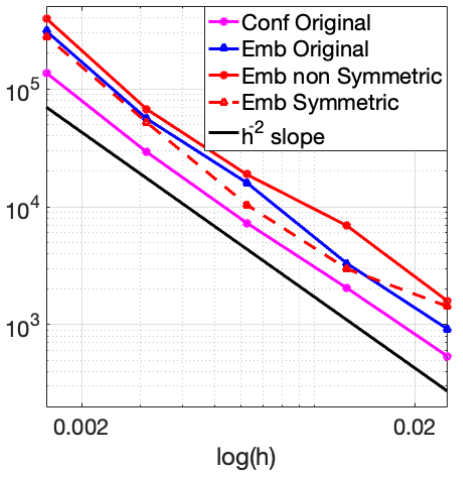

(b) Discontinuous scheme

Figure 8: Matrix condition number - the black line corresponds to the slope $h^{-2}$. Note that the condition number associated with the SBM is not impacted by the element cut ratio. Hence, this figure also shows how the SBM is not affected by the small cut-cell problem.

convergence rates for both continuous and discontinuous discretizations are given in Figure 4(c), for the flux, and Figure 4(d), for the pressure.

The numerical results confirm the expected convergence rates, in the sense that the original formulation has a second-order accurate pressure and a first-order accurate flux, while the enriched pressure formulation yields a thirdorder pressure and a second-order flux.

These convergence rates are similar to the ones obtained in [22] when pressure and velocity are discretized with quadratic and linear interpolation, respectively. However, in the present study, both variables are discretized using piecewise linear interpolation. Therefore, a reduced number of degrees-of-freedom still allows to recover the same accuracy.

\subsubsection{Embedded results}

We consider two boundary-condition configurations: in a first configuration, termed Dirichlet/Dirichlet, Dirichlet boundary conditions are applied on both the inner and outer boundaries (see Fig. 3(b),3(c)); in a second configuration, termed Neumann/Dirichlet, Neumann conditions are applied on the inner boundary while Dirichlet conditions are applied on the outer boundary.

For the Dirichlet/Dirichlet simulations, convergence rates obtained with isotropic permeability for both the CG and DG schemes are shown in Figure 5(a) and 5(d) for the flux and the pressure variables, respectively. The anisotropic 


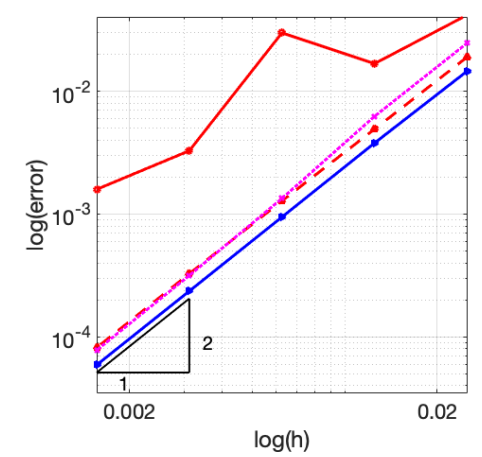

(a) Flux

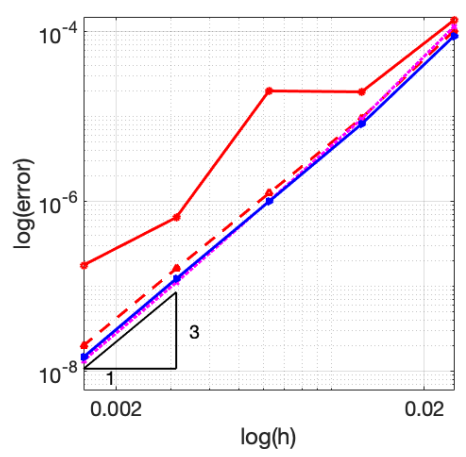

(b) Pressure

Figure 9: Convergence rates: Comparison among various symmetric and non-symmetric formulations. The continuous blue curve corresponds to the symmetric formulation; the continuous red curve corresponds non-symmetric formulation without the "div-div" stabilization or tangential boundary penalty; the dashed red curve corresponds to the non-symmetric formulation with "div-div" stabilization and the dashed magenta curve to the same non-symmetric formulation but with the tangential penalty term instead.

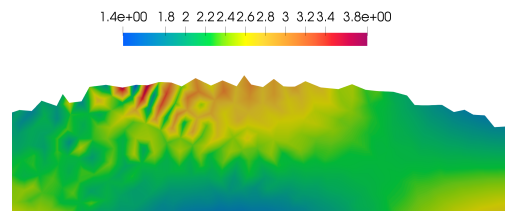

(a) No "div-div" stabilization, no tangential penalty

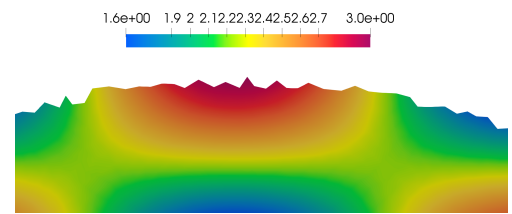

(b) "div-div" stabilization active, no tangential penalty

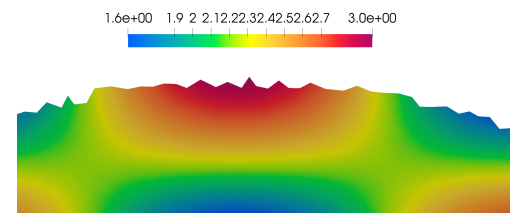

(c) No "div-div" stab, tangential penalty active

Figure 10: Comparison of various non-symmetric formulations. Analysis of spurious modes in the flux.

permeability results are plotted in Figures 5(b) (circular inner boundary) and 5(c) (square inner boundary) for the flux, and in Figure 5(e) (circular inner boundary) and 6(f) (square inner boundary) for the pressure.

The convergence rates corresponding to the Neumann/Dirichlet combination of boundary conditions and isotropic variable permeability are shown in Figure 6(a) for the flux and in Figure 6(d) for the pressure. The corresponding plots for the anisotropic permeability case are given in Figures 6(b) (circular inner boundary), 6(c) (square inner boundary) for the flux and 6(e), 6(f) for the pressure.

The reported numerical results match the expected convergence rates. When the pressure enrichment is activated, the flux is always second-order accurate, while the pressure is third-order accurate for pure Dirichlet boundary conditions and second-order accurate for Neumann boundary conditions. We thus validated the gain of one order of accuracy for both the flux and pressure variables using the pressure enrichment.

For reference, the plots of the solutions (with variable permeability distribution on the surrogate domain) are displayed in Figure 7.

The condition number of the algebraic system associated with the proposed methods is plotted, as a function of the mesh size, in Figure 8. One can see that the use of the pressure enrichment has a minimum influence on the condition number.

\subsubsection{Comparative study among various weak formulations}

This section presents a comparison between the non-symmetric formulation (44) and the symmetric formulation (54). We focus on the CG case, in which the edge penalty term has no beneficial effect.

In section (5.2), we argued that the non-symmetric modification of the schemes requires the use of "div-div" stabilization or some additional tangential penalty. Here, we investigate the different among the following approaches:

- Non-symmetric enrichment with momentum stabilization and no additional stabilization or tangential penalty term; 
- Non-symmetric enrichment with momentum and "div-div" stabilization; no tangential penalty term;

- Non-symmetric enrichment with momentum stabilization and tangential penalty term (with $\alpha_{\tau}=2.0$ );

- Symmetric enrichment with momentum stabilization.

The convergence plots obtained with Dirichlet/Dirichlet combination are displayed in Figure 9 show that the nonsymmetric enrichment with only the momentum stabilization is unstable (see also the spurious modes appearing near the embedded boundary in Figure 10). The addition of either the "div-div" stabilization or the tangential penalty term reestablishes stability in the solution with the expected convergence rate, similar to the one obtained with the symmetric enriched formulation.

\subsection{Patch test cases}

We now tackle the exact representation of polynomial solutions of the same degree of the finite element approximation spaces utilized. For this purpose, we perform tests similar to the ones presented in [36] with discontinuous permeabilities. We consider again an annulus geometry with inner radius $r_{\text {in }}=0.1$ and outer radius $r_{\text {out }}=0.5$, embedded in a disk of radius $R=0.6$. Neumann and Dirichlet conditions are imposed on the inner and outer boundaries, respectively. The computational grid is composed of 2,531 nodes and 4,908 triangles, and the interface $x=0.5$ is meshed explicitly (i.e., as body fitted, see Fig. 11). The permeability distribution is chosen to be $\boldsymbol{\Lambda}=\kappa(x, y) \mathbb{I}$, where

$$
\kappa(x, y)= \begin{cases}\kappa_{1} & \text { if } x \leq 0.5 \\ \kappa_{2} & \text { if } x>0.5\end{cases}
$$

We consider a generalization of the exact solutions considered in [36] to any polynomial degree $r$ :

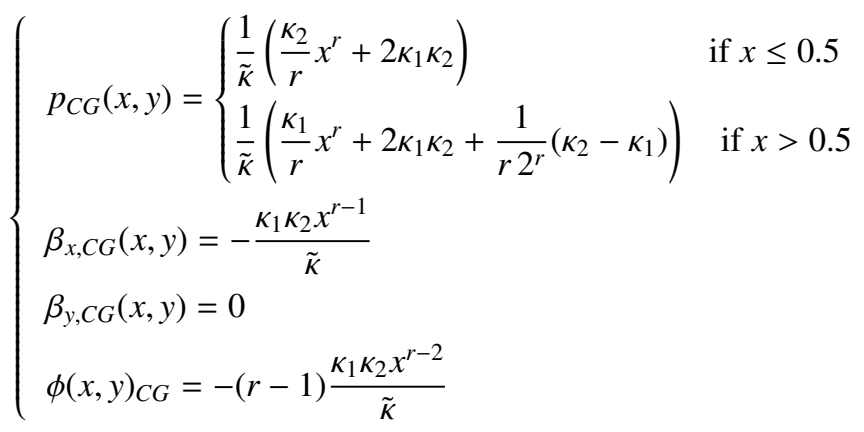

with $\tilde{\kappa}=0.5\left(\kappa_{1}+\kappa_{2}\right)+4 \kappa_{1} \kappa_{2}$, and

$$
\left\{\begin{array}{l}
p_{D G}(x, y)= \begin{cases}1+x^{r}+y^{r} \\
1+\frac{\kappa_{2}-\kappa_{1}}{2^{r} \kappa_{2}}+\frac{\kappa_{1}}{\kappa_{2}} x^{r}+y^{r} & \text { if } x>0.5\end{cases} \\
\beta_{x, D G}(x, y)=-\kappa_{1} r x^{r-1} \\
\beta_{y, D G}(x, y)= \begin{cases}-\kappa_{1} r y^{r-1} & \text { if } x \leq 0.5 \\
-\kappa_{2} r y^{r-1} & \text { if } x>0.5\end{cases} \\
\phi_{D G}(x, y)= \begin{cases}-\kappa_{1} r(r-1)\left(x^{r-2}+y^{r-2}\right) \\
-r(r-1)\left(\kappa_{1} x^{r-2}+\kappa_{2} y^{r-2}\right) & \text { if } x \leq 0.5\end{cases}
\end{array}\right.
$$

Note that in the last two equations of (64)-(65) we define $x^{\alpha}=0$ if $\alpha<0$. Observe also that (64) implies that both the pressure and the flux (gradient) are continuous, while in (65) the flux is discontinuous. Hence, a patch test with (64) can be in principle passed by both DG and CG schemes with pressure enrichment, while (65) can be solved exactly only by the DG scheme.

We perform the simulations for a linear pressure / constant flux $(r=1)$ and quadratic pressure / linear flux $(r=2)$ with and without the enrichment. 


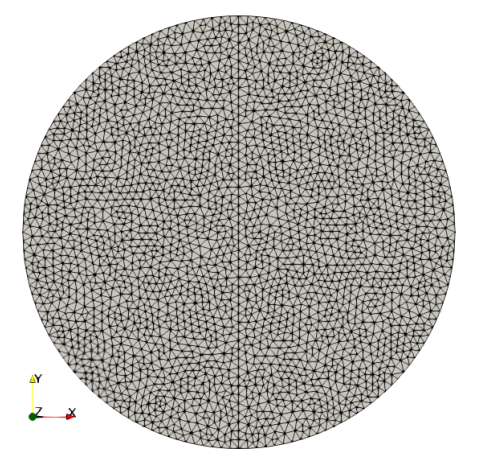

Figure 11: Patch tests mesh.

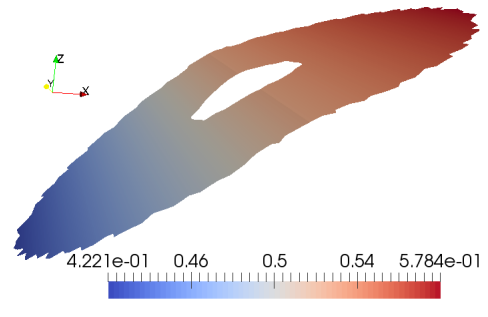

(a) Pressure: Exact

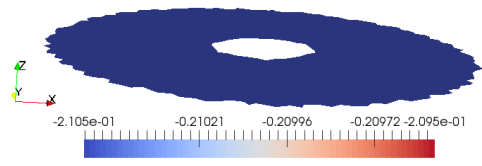

(d) $\beta_{x}$ : Exact

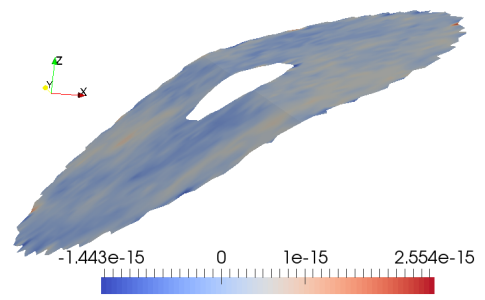

(b) Pressure error: CG

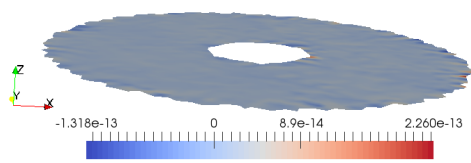

(e) $\beta_{x}$ error: $\mathrm{CG}$

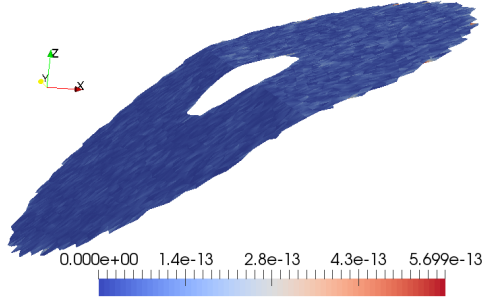

(c) Pressure error: DG

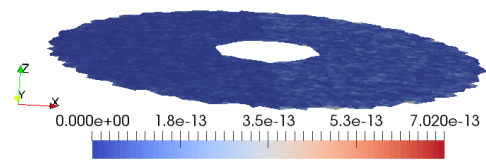

(f) $\beta_{x}$ error: DG

Figure 12: Patch test for linear solution $(r=1)$ : Elevation plots of the pressure and the flux.

For $r=1$ and the exact solution (64), plots of the pressure and one flux component, with their respective errors, are shown in Figure 12, for both the CG and DG schemes. For $r=1$ and the exact solution (65), similar plots are shown in Figure 13, only for the DG scheme. As expected, for the continuous variables, both schemes perfectly match the exact solution. For all these settings, the CG and DG schemes can represent exactly the solutions. However, note that a continuous flux approximation would not work for the exact solution (65), as evident in Figure 14, which shows oscillations near the interface $x=0.5$.

Considering now the case $r=2$ and the continuous exact solution (64), since CG and DG schemes behave identically, we plot in Figure 15 only the results obtained with the continuous formulation along with the errors obtained with both original and enriched schemes. For the discontinuous solution (65), the simulations are only performed with the discontinuous scheme and the results are displayed in Figure 16. It is clearly seen that while the original linear CG and DG schemes do not allow to recover the exact solutions, the enriched formulations perfectly match quadratic solutions (i.e., within machine precision).

\subsection{Domain with a circular impermeable obstruction}

The domain is $\Omega=[0,1] \times[0,1] \backslash \mathscr{C}$ where $\mathscr{C}$ is a circle of radius 0.2 centered in $(0.5,0.5)$. The permeability in the domain is $\boldsymbol{\Lambda}=\mathbb{I}$. On the left and right side we apply Dirichlet conditions with respectively $p=1$ and $p=0$ while all other boundaries are set to slip wall: $\boldsymbol{\beta} \cdot \boldsymbol{n}=0$ (see Fig. 17(a)). As there is no discontinuity in the permeability, the continuous scheme is employed. We compare the solutions for a conformal simulation and one where the inner impermeable obstruction is embedded. The meshes employed are shown in Figures 17(b) and 17(c). Isolines of the 


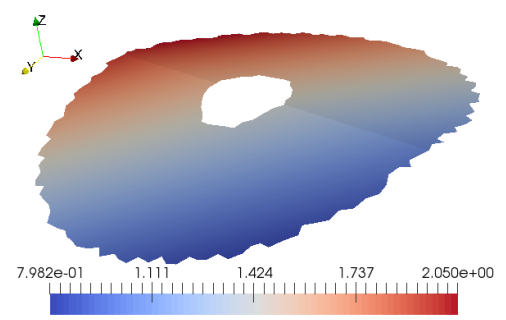

(a) Pressure: Exact

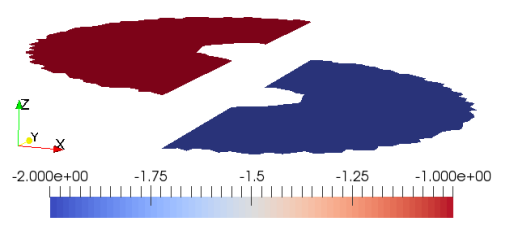

(c) $\beta_{y}$ : Exact

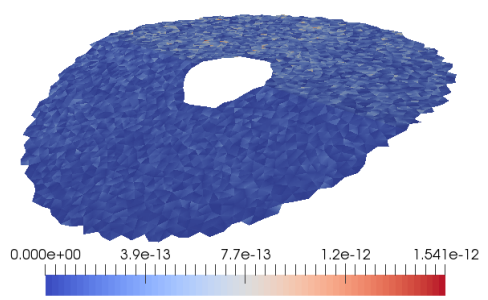

(b) Pressure error

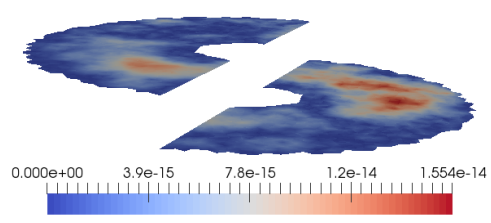

(d) $\beta_{y}$ error

Figure 13: Patch test with DG scheme: Elevation plots of the pressure and the flux.

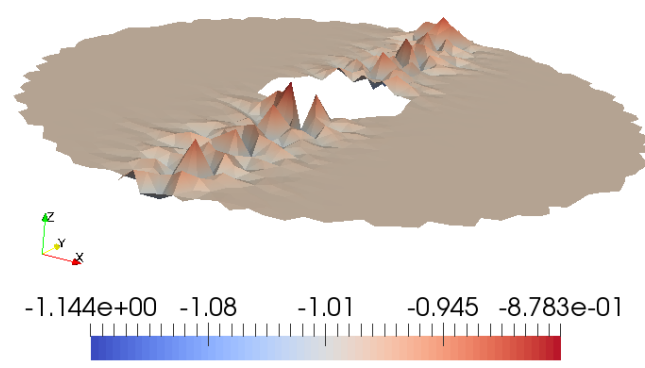

(a) $x$ component

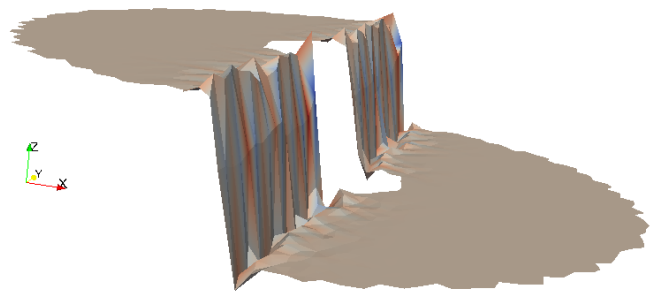

$\begin{array}{lllll}-1.144 e+00 & -1.08 & -1.01 & -0.945 & -8.783 e-01\end{array}$

(b) $y$ component

Figure 14: Flux obtained with CG scheme for the discontinuous exact solution. 


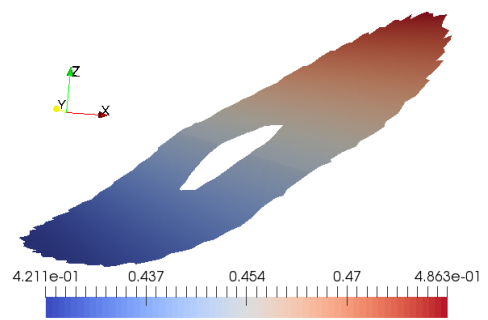

(a) Pressure: Exact

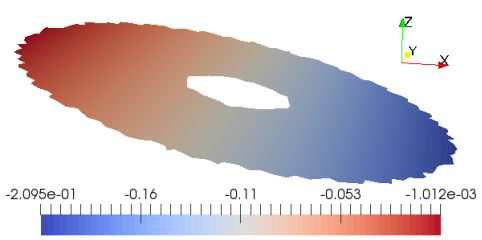

(d) $\beta_{x}$ : Exact

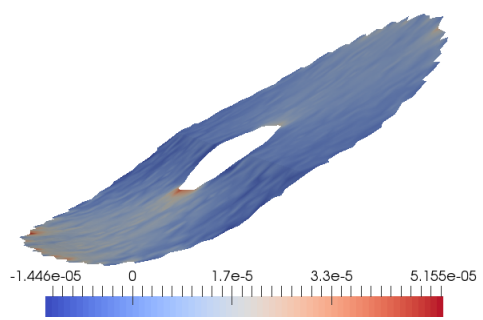

(b) Pressure error: Original scheme

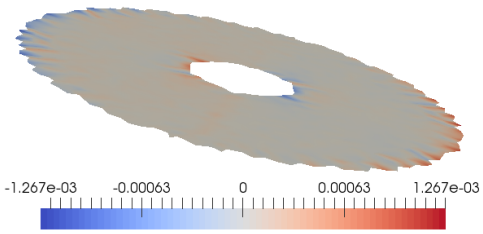

(e) $\beta_{x}$ error: Original scheme

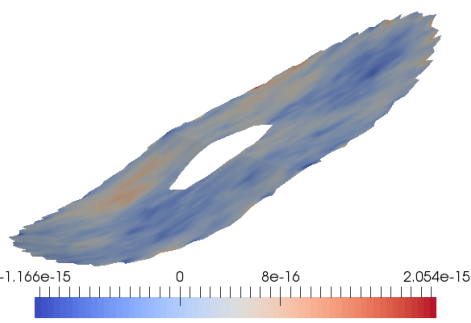

(c) Pressure error: Enriched scheme

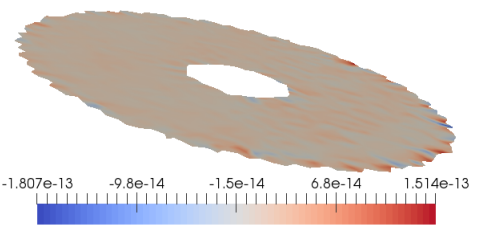

(f) $\beta_{x}$ error: Enriched scheme

Figure 15: Patch test with CG scheme. Elevation plots of the quadratic pressure and linear flux.

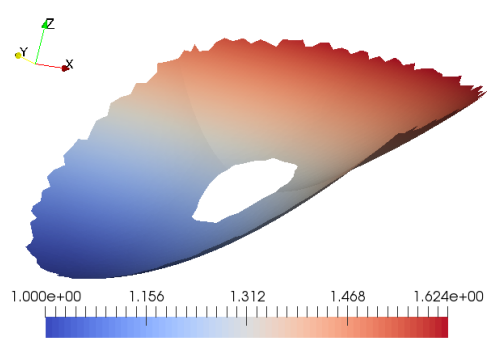

(a) Pressure: Exact

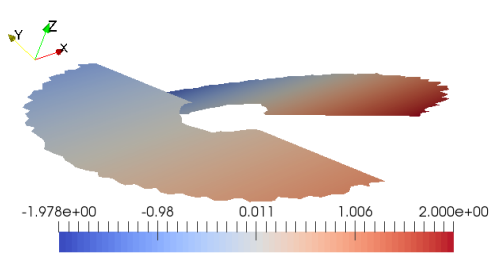

(d) $\beta_{y}$ : Exact

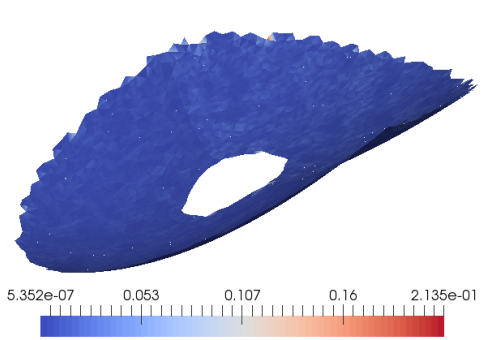

(b) Pressure error: Original scheme

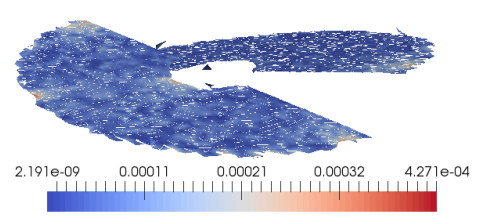

(e) $\beta_{y}$ error: Original scheme

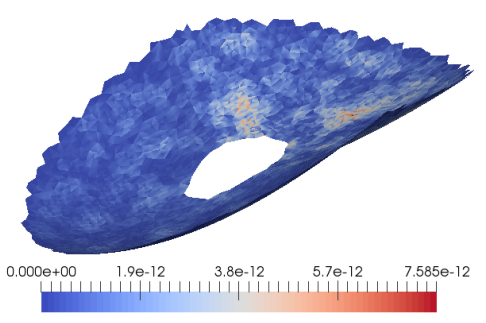

(c) Pressure error: Enriched scheme

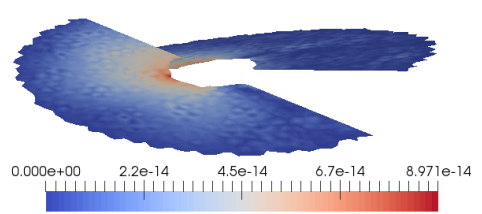

(f) $\beta_{y}$ error: Enriched scheme

Figure 16: Patch test with DG scheme. Elevation plots of the quadratic pressure and linear flux. 


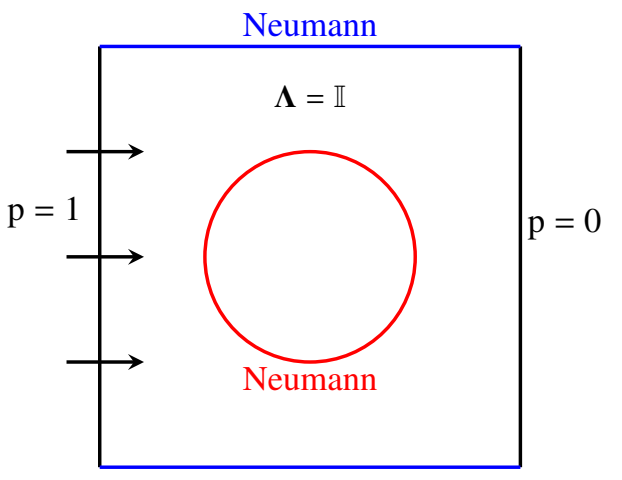

(a) Domain and boundary conditions

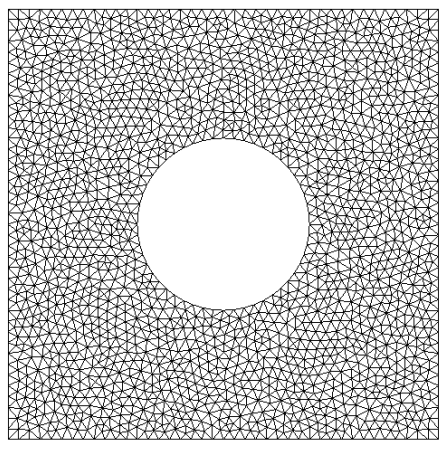

(b) Conformal mesh

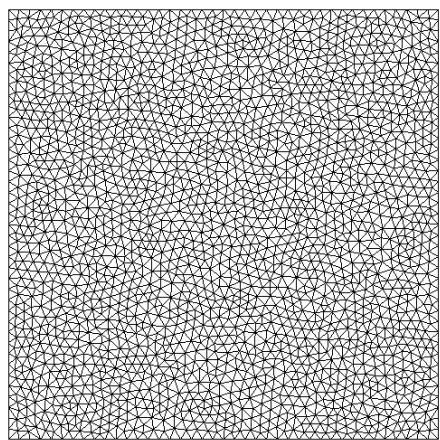

(c) Embedded mesh

Figure 17: Circular impermeable obstruction test. Setup and grids.

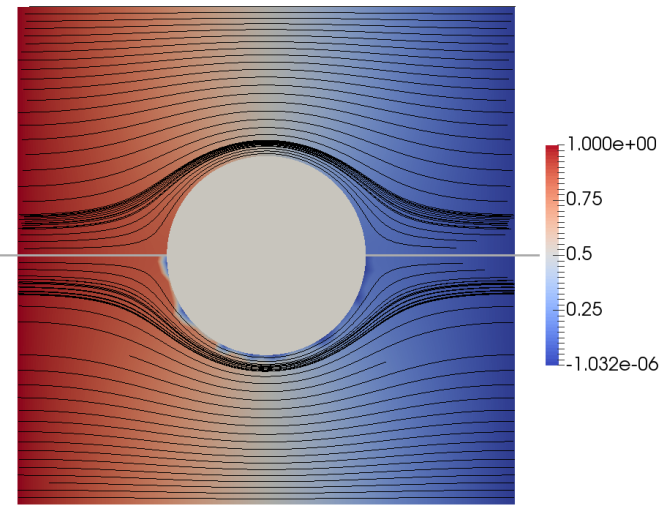

(a) Flux isolines (in black) and pressure. Top half: conformal result. Bottom half: embedded result.

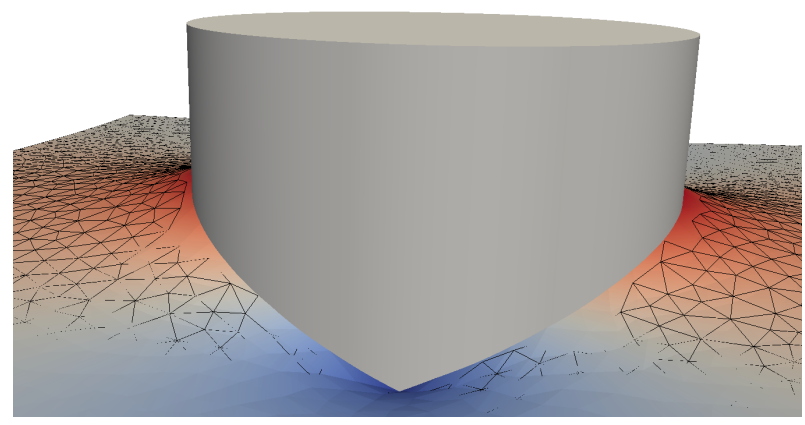

(b) Elevation plots of the flux magnitude: conformal simulation in solid contour; embedded simulations in black wireframe contour

Figure 18: Circular impermeable obstruction test. Comparison between embedded and conformal simulations.

flux and pressure are displayed for both simulations in Figure 18(a) and elevation plots of the flux magnitude are compared in Figure 18(b). The solutions are practically identical.

\subsection{Flow in a domain with a low permeability obstruction}

A square domain $\Omega=[0,1] \times[0,1]$ is divided into two regions. An inner rectangular region $[3 / 8,5 / 8] \times[1 / 4,3 / 4]$ with low permeability $\left(\boldsymbol{\Lambda}=10^{-3} \mathbb{I}\right)$ is surrounded by an area of considerably higher permeability $(\boldsymbol{\Lambda}=\mathbb{I})$. The $p=1$ and $p=0$ are enforced, respectively, on the left and right sides of the domain, and a Neumann condition $\boldsymbol{\beta} \cdot \boldsymbol{n}=0$ is applied on the top and bottom sides (see Fig. 19(a)). For the embedded simulation, the entire domain is embedded in a larger square domain. Since the permeability is discontinuous, the DG scheme is used. Meshes are presented in Figures 19(b) and 19(c). We propose a comparison of the solution obtained with the conformal and embedded simulation in Figure 20. Once again, one can appreciate the similarities between the two results, which validate the discontinuous embedded discretization.

\subsection{A three-dimensional convergence test}

We embed a sphere of radius $r_{\text {in }}=0.2$ in a bigger sphere of radius $r_{\text {out }}=0.5$. The simulations are performed with 6 levels of refinement, from a mesh of 330 nodes and 1,420 tetrahedrons to a mesh of 7,838,897 nodes and 


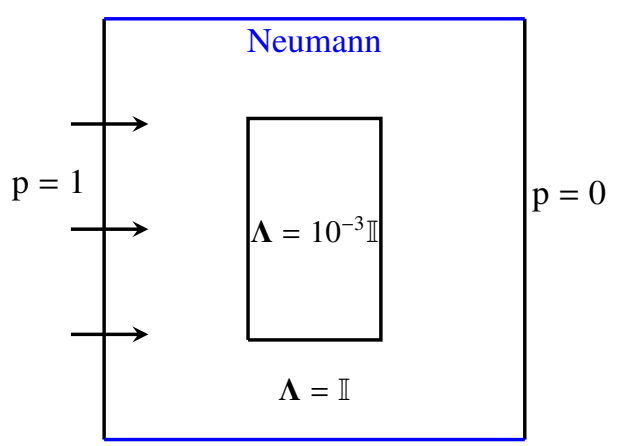

(a) Set up

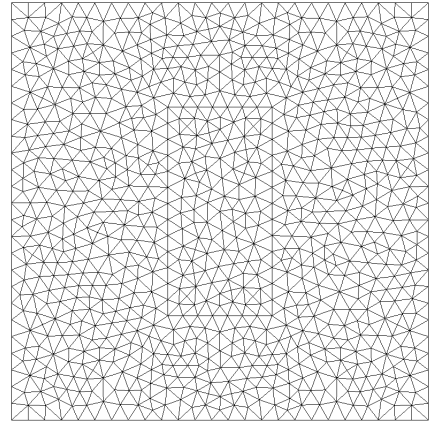

(b) Fitted mesh

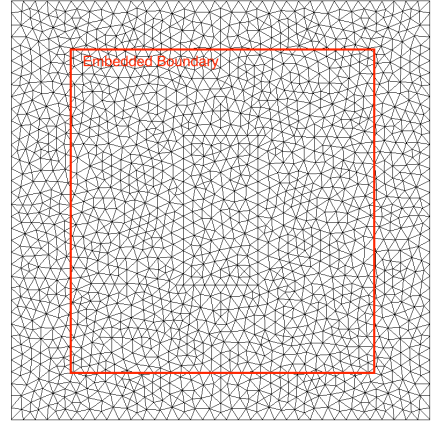

(c) Embedded mesh

Figure 19: Low Permeability obstruction test case.

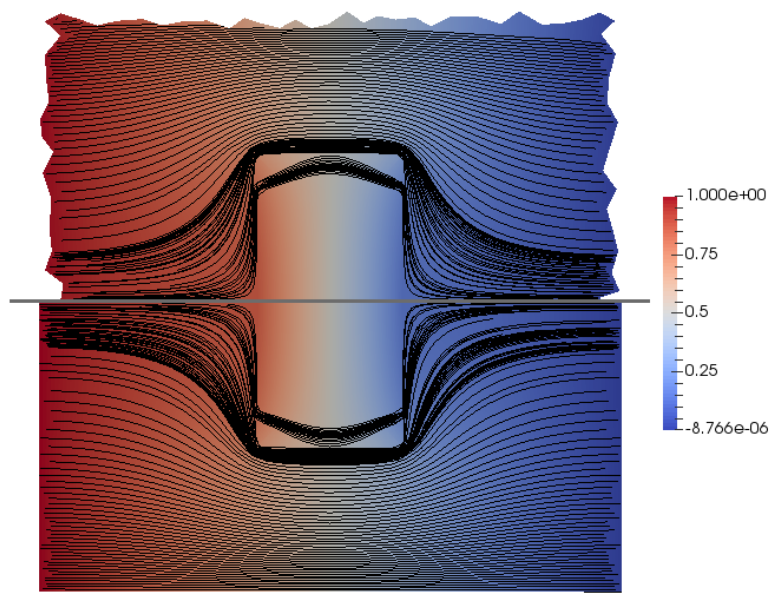

Figure 20: Low permeability obstruction - Comparison of embedded and conformal simulations. Flux isolines on top of pressure contours.

46, 530, 560 tetrahedrons. The exact solution is defined as:

$$
\left\{\begin{array}{l}
\boldsymbol{\beta}(\boldsymbol{x})=-\frac{1}{6}\left\{\begin{array}{l}
\cos (x)(\sinh (z)+\sinh (y))+6 x \\
\cos (y) \sinh (z)+\sin (x) \cosh (y)+2 y \\
\cosh (z)(\sin (y)+\sin (x))-2 z
\end{array}\right\} \\
p(\boldsymbol{x})=\frac{1}{6}\left(\sinh (z)(\sin (y)+\sin (x))+\sin (x) \sinh (y)+3 x^{2}+y^{2}-z^{2}\right) \\
\phi(x)=-1 \\
\boldsymbol{\Lambda}=\mathbb{I}
\end{array}\right.
$$

The convergence rates obtained with and without the pressure enrichment are plotted in Figure 21. There we can appreciate an analogous behavior with respect to the two-dimensional cases, that is, second-order convergence for the flux and pressure in the case of pressure enrichment, and first-order convergence for both fields if no enrichment is provided.

\subsection{Flow past a complicated three-dimensional object}

In order to demonstrate the applicability of the proposed algorithms, we simulate the flow around a complicated object, named "Monkey Trefoil" and already employed in [28] (see Fig. 22). The surface is embedded in a domain of 


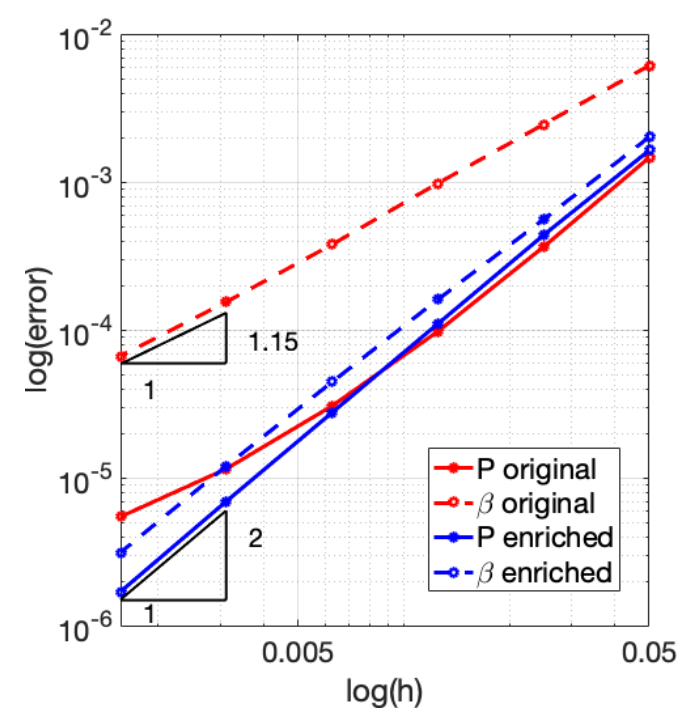

Figure 21: Embedded Neumann 3D simulation: Convergence rates.

size $[-4,4] \times[-2,2] \times[-4,4]$ and meshed with $9,764,152$ tetrahedra. The Dirichlet boundary conditions $p=1$ and $p=0$ are applied at $x=-4$ and $x=4$, respectively. Neumann conditions are applied on the other sides of the domain, as well as on the embedded body. Flux isolines and pressure isosurfaces are shown in Figures 23(a) and 23(b), and demonstrate the robustness of the proposed approach in complex geometry.

\section{Conclusion}

We have developed enhanced embedded mixed finite elements methods for Darcy-type equations that, for linear approximation spaces, deliver second-order error convergence for the primary and gradient variables. In the case when only pure Dirichlet boundary conditions are embedded, the primary variable becomes third-order accurate.

The constructions performed exploit the mixed form of the problem to develop an enrichment of the primary variable. When both test and trial spaces are enriched, all the classical estimates can be generalized in the body fitted case. Extensive numerical simulations has confirmed all the theoretical expectations concerning both accuracy and stability, also when strong discontinuities in the permeability exist.

\section{Acknowledgements}

The first and the last author would like to gratefully acknowledge the support of the U.S. Department of Energy, Office of Science, Advanced Scientific Computing Research under the Early Career Research Program Grant SC0012169, U.S. Army Research Office, under Grant W911NF1810308, and ExxonMobil Upstream Research Company (Houston, TX). This work has also greatly benefitted from the support of the Associated Team HAMSTER between Duke University and the CARDAMOM INRIA team, in Bordeaux (France).

\section{AppendixA. Conformal enriched scheme analysis}

In this appendix, we provide some analysis of the enriched schemes (44) and (54). We start by providing the detailed version of Theorem 1 for the symmetric enrichment. We then provide some additional comments on the non symmetric formulation. Also, we only focus on the conformal case, and refer to [28, 29] for a discussion on the issue of the stability of the SBM approximation. 


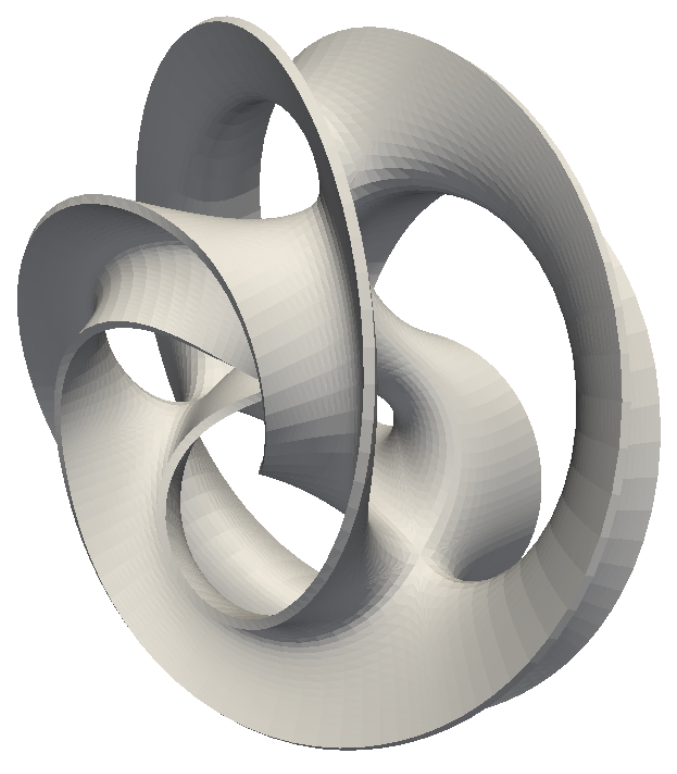

Figure 22: Monkey trefoil geometry.

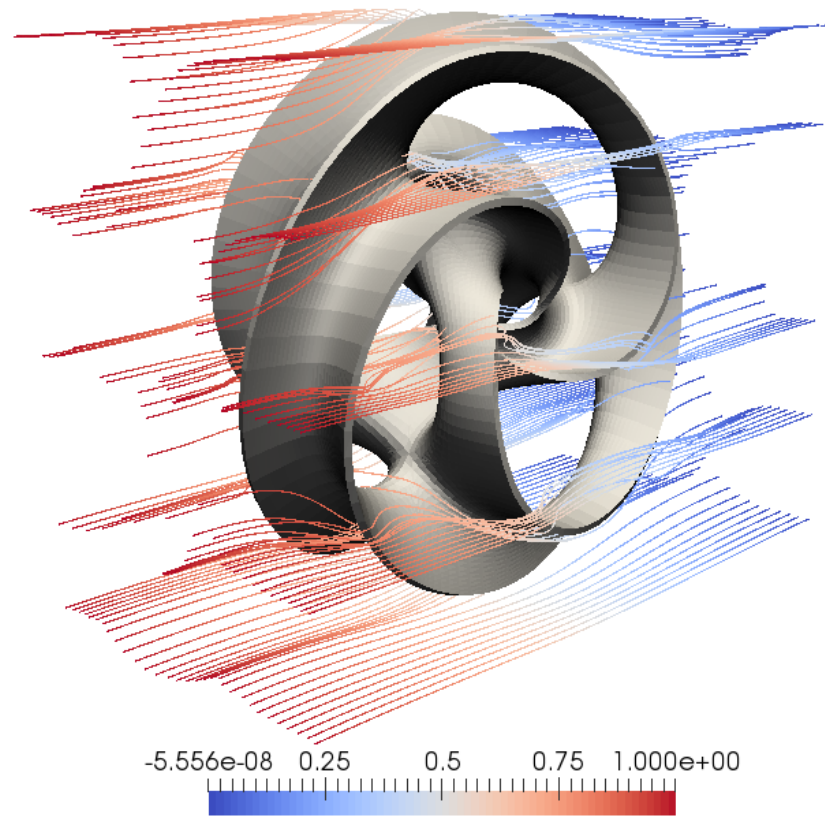

(a) Flux isolines colored by pressure

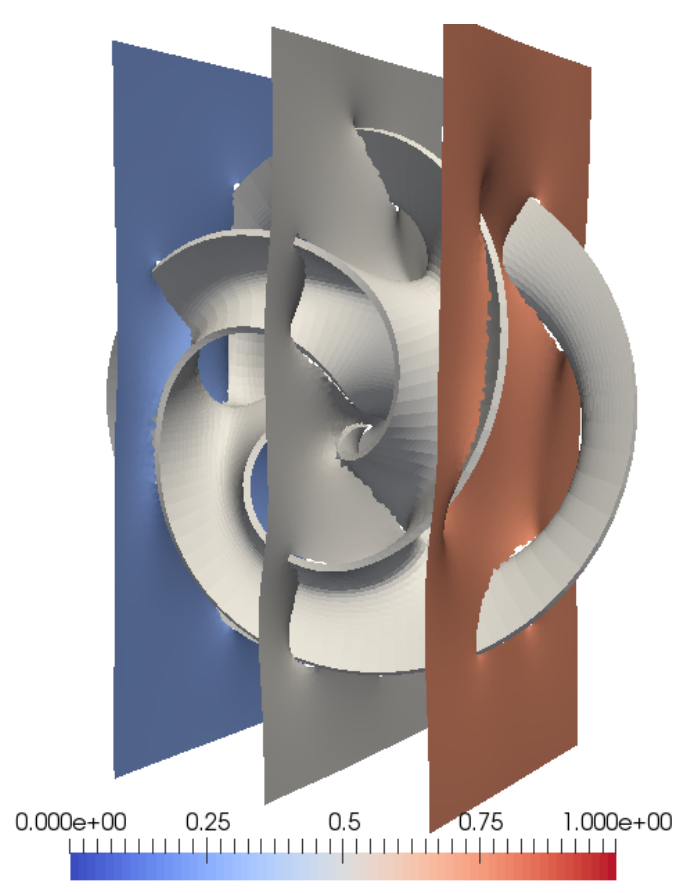

(b) Pressure isosurfaces

Figure 23: Monkey trefoil test. 


\section{AppendixA.1. Symmetric enrichment}

The main idea in the proof of Theorem 1 resides in the fact that the test and trial function spaces for the pressure are identical. Therefore, the analysis of the scheme can be performed in a similar manner to the original continuous and discontinuous schemes (13) proposed in [22, 30].

Proposition 1 (Stability). For all $\beta, p^{*} \in V_{1}(\Omega) \times \mathscr{R}_{1}^{2}\left(V_{1}(\Omega), S_{1}(\Omega)\right.$, the enriched formulation (54) is stable (coercive) in the norm:

$$
\begin{aligned}
\left\|\left(\boldsymbol{\beta}, p^{*}\right)\right\| \|^{2}= & \frac{1}{2}\left(\left\|\boldsymbol{\Lambda}^{-\mathbf{1} / \mathbf{2}} \boldsymbol{\beta}\right\|_{\Omega}^{2}+\left\|\boldsymbol{\Lambda}^{\mathbf{1} / \mathbf{2}} \nabla p^{*}\right\|_{\Omega}^{2}\right) \\
& +\left\|\left(\tilde{\alpha}_{\beta}\|\mathbf{\Lambda}\| / h^{\perp}\right)^{1 / 2} \llbracket p^{*} \rrbracket\right\|_{\mathscr{E}_{i}}^{2}+\left\|\left(\tilde{\alpha}_{\beta}\|\boldsymbol{\Lambda}\| / h^{\perp}\right)^{1 / 2} p^{*}\right\|_{\Gamma_{D}}^{2}+\left\|\left(\tilde{\alpha}_{p} h^{\perp} /\|\mathbf{\Lambda}\|\right)^{1 / 2} \llbracket \boldsymbol{\beta} \rrbracket\right\|^{2}
\end{aligned}
$$

Proof. The proof is immediate, by substitution. For all $\left(\boldsymbol{w}, q^{*}\right) \in \boldsymbol{V}_{1}(\Omega) \times \mathscr{R}_{1}^{2}\left(\boldsymbol{V}_{1}(\Omega) \times S_{1}(\Omega)\right)$ :

$$
\left.B\left(\boldsymbol{w}, q^{*} ; \boldsymbol{w}, q^{*}\right)=\|\left(\boldsymbol{w}, q^{*}\right)\right) \|^{2}
$$

Setting $\boldsymbol{W}=(\boldsymbol{w}, q), \boldsymbol{W}^{*}=\left(\boldsymbol{w}, \mathscr{R}_{1}^{2}(\boldsymbol{w}, q)\right)$, we indicate with $\boldsymbol{V}_{e}=\left(\boldsymbol{\beta}_{e}, p_{e}\right)$ a sufficiently smooth solution. We denote with the symbol $\sim$ an interpolation operator. Hence, $\tilde{\boldsymbol{V}}^{*}$ is the interpolate of the reconstruction (52), and $\tilde{\boldsymbol{V}}^{\mathscr{P} 1}$ and $\tilde{\boldsymbol{V}}^{\mathscr{P} 2}$ are, respectively, the $\mathscr{P}^{1}$ and $\mathscr{P}^{2}$ Lagrange interpolants. We then decompose the error as

$$
\boldsymbol{E}=\boldsymbol{V}^{*}-\boldsymbol{V}_{e}=\overbrace{\boldsymbol{V}^{*}-\tilde{\boldsymbol{V}}_{e}^{*}}^{\boldsymbol{E}^{*}}+\overbrace{\tilde{\boldsymbol{V}}_{e}^{*}-\boldsymbol{V}}^{\boldsymbol{H}_{e}^{*}}
$$

where $\boldsymbol{E}=\left(\boldsymbol{\beta}-\boldsymbol{\beta}_{e} ; p^{*}-p_{e}\right), \boldsymbol{E}^{*}=\left(\boldsymbol{\beta}-\tilde{\boldsymbol{\beta}}_{e}^{*} ; p^{*}-\tilde{p}_{e}^{*}\right), \boldsymbol{H}^{*}=\left(\tilde{\boldsymbol{\beta}}_{e}^{*}-\boldsymbol{\beta}_{e} ; \tilde{p}_{e}^{*}-p_{e}\right)$. Consistency, or better, Galerkin orthogonality is immediate to prove, once we observe that $B_{d}\left(\boldsymbol{W}^{*}, \boldsymbol{V}_{e}\right)=L_{d}\left(\boldsymbol{W}^{*}\right)$ :

Proposition 2 (Consistency). For all $\boldsymbol{W}^{*} \in \boldsymbol{V}^{1}(\Omega) \times \mathscr{R}_{1}^{2}\left(\boldsymbol{V}_{1}(\Omega), S_{1}(\Omega)\right)$ :

$$
B_{d}\left(\boldsymbol{W}^{*}, \boldsymbol{E}\right)=0
$$

Finally, we can focus on the accuracy of the scheme. Using the decomposition of the error between the approximation and truncation errors, the following theorem holds, for the "classical" non enriched formulation (see [30] for detailed proof):

Theorem 2. For $(\boldsymbol{\beta}, p) \in \boldsymbol{V}^{l}(\Omega) \times S^{k}(\Omega)$ :

$$
\|\boldsymbol{E}|\|\leq C|\|\boldsymbol{H}\||
$$

with

$$
\left\||\boldsymbol{H} \|| \leq C_{1} h^{k+1}|\boldsymbol{\beta}|_{k+1}+C_{2} h^{l}|p|_{l+1}\right.
$$

and $|.|_{s}$ the sth Sobolev seminorm.

In our case, the same consistency/stability argument that yields the proof of Theorem 2 can be followed, step-bystep, to obtain $\|\boldsymbol{E}\|\left|\leq C\left\|\boldsymbol{H}^{*} \mid\right\|\right.$. We then seek an upper bound of $\boldsymbol{H}^{*}$. To this purpose, we introduce the vector of the $\mathscr{P}^{1}$ and $\mathscr{P}^{2}$ interpolates of the flux and pressure $\tilde{\boldsymbol{V}}_{e}=\left(\tilde{\boldsymbol{\beta}}_{e}^{\mathscr{P}^{1}}, \tilde{p}_{e}^{\mathscr{P}^{2}}\right)$ and we decompose $\boldsymbol{H}^{*}$ as:

$$
\boldsymbol{H}^{*}=\tilde{\boldsymbol{V}}_{e}^{*}-\boldsymbol{V}_{e}=\overbrace{\boldsymbol{V}_{e}^{*}-\tilde{\boldsymbol{V}}_{e}}^{\boldsymbol{H}^{*, 2}}+\overbrace{\tilde{\boldsymbol{V}}_{e}-\boldsymbol{V}_{e}}^{\tilde{\boldsymbol{H}}}
$$

where $\tilde{\boldsymbol{H}}=\left(\boldsymbol{\beta}^{\mathscr{P}^{1}}-\boldsymbol{\beta}_{e}, \tilde{p}^{\mathscr{P}^{2}}-p_{e}\right)$ the $\mathscr{P}^{1}$ and $\mathscr{P}^{2}$ Lagrange interpolation error of respectively the flux and the pressure, satisfying (A.6)

$$
\left.\|\tilde{\boldsymbol{H}}\|\left|\leq C_{1} h^{2}\right| \boldsymbol{\beta}\right|_{2}+C_{2} h^{2}|p|_{3}
$$

and $\boldsymbol{H}^{*, 2}=\left(\tilde{\boldsymbol{\beta}}^{*}-\tilde{\boldsymbol{\beta}}^{\mathscr{P}^{1}}, \tilde{p}^{*}-\tilde{p}^{\mathscr{P}^{2}}\right)=\left(\mathbf{0}, \tilde{p}^{*}-\tilde{p}^{2}\right)$ represents the error between the quadratic reconstruction of the pressure with (52) and the $\mathscr{P}^{2}$ one, as the mapping (52) approximate linearly the flux. We then have the following 
Lemma 1. The norm of the error between the two quadratic interpolations $\boldsymbol{H}^{*, 2}$ is bounded by the interpolation error on the flux $\beta$ :

$$
\|\left|\boldsymbol{H}^{*, 2}\right||| \leq C_{3} h^{2}|\boldsymbol{\beta}|_{2}
$$

Proof. Let first recall some useful inequalities. The trace inequality for a simplex $K$ of the mesh writes:

$$
\|q\|_{\partial K}^{2} \leq C_{T}\left(h^{-1}\|q\|_{K}^{2}+h\|\nabla q\|_{K}^{2}\right)
$$

where $C_{T}$ is a constant. In addition, assuming the mesh regular enough, we have

$$
\|\nabla q\|_{K} \leq C_{i n v} h^{-1}\|q\|_{K}
$$

and there exists constants $\tilde{C}_{1}$ and $\tilde{C}_{2}$ such that, for all edges on the mesh $\boldsymbol{e}_{i \rightarrow j}$,

$$
\tilde{C}_{1} h \leq\left\|\boldsymbol{e}_{i \rightarrow j}\right\| \leq \tilde{C}_{2} h
$$

Now, from (A.11), we can provide a stronger form of the trace inequality (A.10) reading:

$$
\|q\|_{\partial K}^{2} \leq C_{T}^{\prime} h^{-1}\|q\|_{K}^{2}
$$

Then, by definition, $\boldsymbol{H}^{*, 2}=\left(\mathbf{0}, \tilde{p}^{*}-\tilde{p}^{\mathscr{P}^{2}}\right)$ and thus we have:

$$
\|\| \boldsymbol{H}^{*, 2}\|\|_{\Omega}^{2}=\frac{1}{2}\left\|\boldsymbol{\Lambda}^{1 / 2} \nabla\left(\tilde{p}^{*}-\tilde{p}^{\mathscr{P} 2}\right)\right\|_{\Omega}^{2}+\left\|\left(\tilde{\alpha}_{\beta} \| \boldsymbol{\Lambda} / h^{\perp}\right)^{1 / 2} \llbracket \tilde{p}^{*}-\tilde{p}^{\mathscr{P}^{2}} \rrbracket\right\|_{\mathscr{E}}^{2}
$$

where $\mathscr{E}=\mathscr{E}_{i} \cup \Gamma_{D}$. For every simplex $K$ we have, by definition (42)

$$
\tilde{p}^{*}=\sum_{i, \text { node }} p_{i} \varphi_{i}^{\mathscr{P}^{2}}+\sum_{m, e d g e}\left(\frac{p_{i}+p_{j}}{2}+\frac{1}{8}\left(\boldsymbol{\Lambda}_{i} \boldsymbol{\beta}_{i}-\boldsymbol{\Lambda}_{j} \boldsymbol{\beta}_{j}\right) \cdot \boldsymbol{e}_{i \rightarrow j}\right) \varphi_{m}^{\mathscr{P}^{2}}
$$

and we can approximate the mid-edge values $\tilde{p}_{m}^{\mathscr{P}^{2}}$ using the same Taylor expansion as in Section 5.1 and define:

$$
\tilde{p}^{\mathscr{P}^{2}}=\sum_{i, \text { node }} p_{i} \varphi_{i}^{\mathscr{P}^{2}}+\sum_{m, \text { edge }} \tilde{p}_{m}^{\mathscr{P}^{2}} \varphi_{m}^{\mathscr{P}^{2}}
$$

where

$$
\tilde{p}_{m}^{\mathscr{P}^{2}}=\frac{p_{i}+p_{j}}{2}-\frac{1}{4}\left(\boldsymbol{\Lambda}_{i} \boldsymbol{\beta}_{i}-\boldsymbol{\Lambda}_{j} \boldsymbol{\beta}_{j}\right)+\frac{1}{16} \boldsymbol{e}_{i \rightarrow j}\left(\mathscr{H}_{i}+\mathscr{H}_{j}\right) \boldsymbol{e}_{i \rightarrow j}+\mathscr{O}\left(h^{3}\right)
$$

This allows to define the difference in pressure interpolations as:

$$
\tilde{p}_{m}^{*}-\tilde{p}_{m}^{\mathscr{P}^{2}}=\frac{1}{8}\left(\left(\boldsymbol{\Lambda}_{j} \boldsymbol{\beta}_{i}-\boldsymbol{\Lambda}_{i} \boldsymbol{\beta}_{j}\right)+\mathscr{H}_{i} \boldsymbol{e}_{i \rightarrow j}+\left(\boldsymbol{\Lambda}_{j} \boldsymbol{\beta}_{i}-\boldsymbol{\Lambda}_{i} \boldsymbol{\beta}_{j}\right)+\mathscr{H}_{j} \boldsymbol{e}_{i \rightarrow j}\right) \cdot \boldsymbol{e}_{i \rightarrow j}
$$

which can be written using the Taylor expansion with Lagrange's reminder (see remark 4):

$$
\tilde{p}_{m}^{*}-\tilde{p}_{m}^{\mathscr{P}^{2}}=\frac{1}{8}\left(\boldsymbol{e}_{i \rightarrow j}^{T} \nabla^{2} \beta(\boldsymbol{c}) \boldsymbol{e}_{i \rightarrow j}\right) \cdot \boldsymbol{e}_{i \rightarrow j}
$$

and thus:

$$
\tilde{p}^{*}(\boldsymbol{x})-\tilde{p}^{\mathscr{P}^{2}}(\boldsymbol{x})=\sum_{m, e d g e} \frac{1}{8}\left(\boldsymbol{e}_{i \rightarrow j}^{T} \boldsymbol{\nabla}^{2} \beta(\boldsymbol{c}) \boldsymbol{e}_{i \rightarrow j}\right) \cdot \boldsymbol{e}_{i \rightarrow j} \varphi_{m}(\boldsymbol{x})
$$


We can now focus on each term of (A.14).

$$
\begin{aligned}
\frac{1}{2}\left\|\boldsymbol{\Lambda}^{1 / 2} \boldsymbol{\nabla}\left(\tilde{p}-\tilde{p}^{\mathscr{P}^{2}}\right)\right\|_{\Omega}^{2} & \leq \frac{C_{i n v}}{2 h^{2}} \sup _{\boldsymbol{x}}\|\boldsymbol{\Lambda}\| \sum_{K}\left\|\tilde{p}-\tilde{p}^{\mathscr{P}^{2}}\right\|_{K}^{2} \\
& \leq \frac{C_{i n v}}{2 h^{2}} \sup _{\boldsymbol{x}}\|\boldsymbol{\Lambda}\| \sum_{K} \int_{K}\left|\tilde{p}^{*}-\tilde{p}^{\mathscr{P}^{2}}\right| \\
& \leq \frac{C_{i n v}}{2 h^{2}} \sup _{\boldsymbol{x}}\|\boldsymbol{\Lambda}\| \sum_{K} \int_{K} \sum_{m, e d g e} \frac{1}{8}\left|\left(\boldsymbol{e}_{i \rightarrow j}^{T} \boldsymbol{\nabla}^{2} \beta(\boldsymbol{c}) \boldsymbol{e}_{i \rightarrow j}\right) \cdot \boldsymbol{e}_{i \rightarrow j} \varphi_{m}(\boldsymbol{x})\right|^{2} \\
& \leq \frac{C_{i n v} \tilde{C}_{2} h^{4}}{2} \sup _{\boldsymbol{x}}\|\boldsymbol{\Lambda}\| \sum_{K} \int_{K} \sum_{m, e d g e} \frac{1}{8}\left|\left(\frac{\boldsymbol{e}_{i \rightarrow j}^{T}}{\left\|\boldsymbol{e}_{i \rightarrow j}\right\|} \nabla^{2} \beta(\boldsymbol{c}) \frac{\boldsymbol{e}_{i \rightarrow j}}{\left\|\boldsymbol{e}_{i \rightarrow j}\right\|}\right) \cdot \frac{\boldsymbol{e}_{i \rightarrow j}}{\left\|\boldsymbol{e}_{i \rightarrow j}\right\|} \varphi_{m}(\boldsymbol{x})\right|^{2}
\end{aligned}
$$

and we can get:

$$
\frac{1}{2}\left\|\boldsymbol{\Lambda}^{1 / 2} \nabla\left(\tilde{p}-\tilde{p}^{\mathscr{P}^{2}}\right)\right\|_{\Omega} \leq C_{3} h^{4}|\boldsymbol{\beta}|_{2}
$$

Denoting $e_{p}=\tilde{p}^{*}-\tilde{p}^{\mathscr{P}^{2}}$, an identical process can bound the error on edges:

$$
\begin{aligned}
& \left\|\left(\tilde{\alpha}_{\beta}\|\mathbf{\Lambda}\| / h^{\perp}\right)^{1 / 2} \llbracket e_{p} \rrbracket\right\|_{\mathscr{E}_{i}}^{2} \\
& +\left\|\left(\tilde{\alpha}_{\beta}\|\mathbf{\Lambda}\| / h^{\perp}\right)^{1 / 2} \llbracket e_{p} \rrbracket\right\|_{\Gamma_{D}}^{2} \leq \frac{\tilde{\alpha}_{\beta}}{h} \sup _{\boldsymbol{x}}\|\boldsymbol{\Lambda}\|\left(\int_{\mathscr{E}_{i}}\left|e_{p}\right|_{+}^{2}+\left|e_{p}\right|_{-}^{2}+\int_{\Gamma_{D}}\left|e_{p}\right|^{2}\right) \\
& \leq \frac{\tilde{\alpha}_{\beta}}{h} \sup _{\boldsymbol{x}}\|\boldsymbol{\Lambda}\| \sum_{K}\left\|p-p^{*}\right\|_{\partial K}^{2} \\
& \leq \frac{\tilde{\alpha}_{\beta} C_{T}^{\prime}}{h^{2}} \sup _{\boldsymbol{x}}\|\boldsymbol{\Lambda}\| \sum_{K}\left\|p-p^{*}\right\|_{K}^{2} \\
& \leq \tilde{\alpha}_{\beta} C_{T}^{\prime} \tilde{C}_{2} h^{4} \sup _{\boldsymbol{x}}\|\boldsymbol{\Lambda}\| \sum_{K} \int_{K} \sum_{m, e d g e} \frac{1}{8}\left|\left(\frac{\boldsymbol{e}_{i \rightarrow j}^{T}}{\left\|\boldsymbol{e}_{i \rightarrow j}\right\|} \nabla^{2} \beta(\boldsymbol{c}) \frac{\boldsymbol{e}_{i \rightarrow j}}{\left\|\boldsymbol{e}_{i \rightarrow j}\right\|}\right) \cdot \frac{\boldsymbol{e}_{i \rightarrow j}}{\left\|\boldsymbol{e}_{i \rightarrow j}\right\|} \varphi_{m}(\boldsymbol{x})\right|^{2}
\end{aligned}
$$

and thus:

$$
\left\|\left(\tilde{\alpha}_{\beta}\|\boldsymbol{\Lambda}\| / h^{\perp}\right)^{1 / 2} \llbracket e_{p} \rrbracket\right\|_{\mathscr{E}} \leq C_{4} h^{2}|\boldsymbol{\beta}|_{2}
$$

Combining (A.22) and (A.24), we have the following final upper bound:

$$
\left.\left\|\left|\boldsymbol{H}^{*, 2}\right|\right\|\left|\leq\left(C_{3}+C_{4}\right) h^{2}\right| \boldsymbol{\beta}\right|_{2}
$$

Thus, the estimate for the interpolation error reads:

Proposition 3 (Error estimate). The scheme (54) is accurate with the error estimate:

$$
\left\|\boldsymbol{E}|\|| \leq \tilde{C}_{1} h^{2}|\boldsymbol{\beta}|_{2}+\tilde{C}_{2} h^{2}|p|_{3}\right.
$$

Proof. By a consistency/stability argument, we obtain a similar inequality to the case of the scheme without enrichment, namely $\left\|\boldsymbol{E}\left|\|\leq C \mid\| \boldsymbol{H}^{*} \|\right.\right.$. Then, using the triangular inequality on (A.7), as a direct consequence of Theorem 2 and Lemma 1, we finally obtain:

$$
\||\boldsymbol{E}|\| \leq C\left(\left(C_{1}+C_{4}\right) h^{2}|\boldsymbol{\beta}|_{2}+C_{2} h^{2}|p|_{3}\right)
$$

\section{AppendixA.2. Non-symmetric enrichment}

As explained in Section 5.2, for the non symmetric scheme, the solution (trial) space $V^{1}(\Omega) \times \mathscr{R}_{1}^{2}\left(S^{1}(\Omega), V^{1}(\Omega)\right)$ is not the same as the test space $V^{1}(\Omega) \times S^{1}(\Omega)$. Note that both $S^{1}(\Omega)$ and $\mathscr{R}_{1}^{2}\left(S^{l}(\Omega), V^{1}(\Omega)\right.$ ) are however sub-spaces of $H^{1}(\Omega)$, which is the infinite dimensional pressure space for the problem under consideration. 
Thus, the stability cannot be proven with same coercivity argument as for the symmetric formulation, but an argument can be provided regarding the consistency and error of consistency.

Consistency can be proved in a very similar manner to case of the symmetric formulation. If $\left(\boldsymbol{\beta}_{e}, p_{e}\right)$ are smooth fields verifying exactly the PDE and the boundary conditions, one can readily show that

$$
0=B_{d}\left(\boldsymbol{w}, q ; \boldsymbol{\beta}-\boldsymbol{\beta}_{e}, \mathscr{R}_{1}^{2}(\boldsymbol{\beta}, p)-p_{e}\right)=B_{d}\left(\boldsymbol{w}, q ; \boldsymbol{\beta}-\boldsymbol{\beta}_{e}, p^{*}-p_{e}\right)
$$

Error analysis. As before, the global error can be split into the approximation and the truncation error. Note that the consistency/orthogonality relation still implies

$$
B_{d}\left(\boldsymbol{W}, \boldsymbol{E}^{*}\right)+B_{d}\left(\boldsymbol{W}, \boldsymbol{H}^{*}\right)=0
$$

And we focus on the consistency error $\left|B_{d}\left(\boldsymbol{W}, \boldsymbol{E}^{*}\right)\right|$, as shown in [1,2]. Using the continuity of $B_{d}$ provides an estimate of the consistency error, namely,

$$
\left|B_{d}\left(\boldsymbol{W}, \boldsymbol{E}^{*}\right)\right| \leq C_{\text {cont }}\|\boldsymbol{W}\|\left\|\boldsymbol{H}^{*}\right\|
$$

where the norms on the right hand side are the natural norms in $H^{\mathrm{div}} \times H^{1}$, and we have:

$$
\|\boldsymbol{H}\|_{\Omega}^{2}=\|\tilde{\boldsymbol{\beta}}-\tilde{\boldsymbol{\beta}}\|_{\Omega}^{2}+\|\boldsymbol{\nabla} \cdot(\tilde{\boldsymbol{\beta}}-\boldsymbol{\beta})\|_{\Omega}^{2}+\|\tilde{p}-p\|_{\Omega}^{2}+\|\boldsymbol{\nabla}(\tilde{p}-p)\|_{\Omega}^{2}
$$

Here $\tilde{p}$ represents the interpolation with or without the enrichment. We then have, using inverse inequality (A.11),

$$
\left|B_{d}(\boldsymbol{W}, \boldsymbol{E})\right|^{2} \leq C_{\text {cont }}^{2}\|\boldsymbol{W}\|_{\infty}^{2}\left(C_{i n v, 1} h^{-2}\|\tilde{\boldsymbol{\beta}}-\boldsymbol{\beta}\|_{\Omega}^{2}+C_{i n v, 2} h^{-2}\|\tilde{p}-p\|_{\Omega}^{2}\right)
$$

Here the interpolation error can be used with $\tilde{p}=\tilde{p}^{\mathscr{P} 1}$ for the original formulation

$$
\left|B_{d}(\boldsymbol{W}, \boldsymbol{E})\right| \leq C_{\text {cont }}\|\boldsymbol{W}\|_{\infty}\left(C_{1} h|\boldsymbol{\beta}|_{2}+C_{2} h|p|_{2}\right)
$$

and $\tilde{p}=\tilde{p}^{*}$ for the non symmetric enrichment. Using the same decomposition as for the symmetric formulation, $\left\|\tilde{p}^{*}-p\right\|_{\Omega} \leq\left\|\tilde{p}^{*}-\tilde{p}^{\mathscr{P}^{2}}\right\|_{\Omega}^{2}+\left\|\tilde{p}^{\mathscr{P}^{2}}-p\right\|_{\Omega}^{2}$, and we end up with:

$$
\left|B_{d}\left(\boldsymbol{W}, \boldsymbol{E}^{*}\right)\right| \leq C_{\text {cont }}\|\boldsymbol{W}\|_{\infty}\left(C_{1} h|\boldsymbol{\beta}|_{2}+C_{2} h^{2}|\beta|_{2}+C_{3} h^{2}|p|_{2}\right)
$$

We can note that although the final estimate is $\mathscr{O}(h)$, due to the divergence part in the classical $H^{d i v}$ norm, the consistency error estimates coming from the pressure are improved when switching from the non enriched to the enriched scheme.

\section{References}

[1] R. Abgrall and M. Ricchiuto. High order methods for CFD. In Encyclopedia of Computational Mechanics. John Wiley \& Sons, Ltd, 2017.

[2] R. Abgrall and P. L. Roe. High order fluctuation schemes on triangular meshes. Journal of Scientific Computing, 19(1):3-36, Dec 2003.

[3] D. Arnold, F. Brezzi, B. Cockburn, and L. Marini. Unified analysis of discontinuous galerkin methods for elliptic problems. SIAM Journal on Numerical Analysis, 39(5):1749-1779, 2002.

[4] Douglas N Arnold. An interior penalty finite element method with discontinuous elements. SIAM journal on numerical analysis, 19(4):742$760,1982$.

[5] Santiago Badia and Ramon Codina. Stabilized continuous and discontinuous galerkin techniques for darcy flow. Computer Methods in Applied Mechanics and Engineering, 199(25):1654 - 1667, 2010.

[6] Laure Billon, Youssef Mesri, and Elie Hachem. Anisotropic boundary layer mesh generation for immersed complex geometries. Engineering with Computers, 33(2):249-260, Apr 2017.

[7] Erik Burman. Ghost penalty. Comptes Rendus Mathematique, 348(21-22):1217-1220, 2010.

[8] Erik Burman and Peter Hansbo. Fictitious domain methods using cut elements: Iii. a stabilized nitsche method for stokes' problem. ESAIM: Mathematical Modelling and Numerical Analysis, 48(3):859-874, 2014.

[9] D. Caraeni and L. Fuchs. Compact third-order multidimensional upwind scheme for navier-stokes simulations. Theoret Comput Fluid Dynamics, 15:373-401, 2002.

[10] D. K. Clarke, H. A. Hassan, and M. D. Salas. Euler calculations for multielement airfoils using cartesian grids. AIAA Journal, 24(3):353-358, 1986.

[11] B. Cockburn, B. Dong, J. Guzmán, M. Restelli, and R. Sacco. A hybridizable discontinuous galerkin method for steady-state convectiondiffusion-reaction problems. SIAM Journal on Scientific Computing, 31(5):3827-3846, 2009. 
[12] Bernardo Cockburn, George E. Karniadakis, and Chi-Wang Shu. Discontinuous Galerkin Methods: Theory, Computation and Applications. Springer, 2000

[13] Jim Douglas and Todd Dupont. Interior penalty procedures for elliptic and parabolic galerkin methods. In R. Glowinski and J. L. Lions, editors, Computing Methods in Applied Sciences, pages 207-216, Berlin, Heidelberg, 1976. Springer Berlin Heidelberg.

[14] Jim Douglas, Mary Fanett Wheeler, Bruce L. Darlow, and Richard P. Kendall. Self-adaptive finite element simulation of miscible displacement in porous media. Computer Methods in Applied Mechanics and Engineering, 47(1):131 - 159, 1984. Special Issue on Oil Reservoir Simulation.

[15] Alexander Düster, Jamshid Parvizian, Zhengxiong Yang, and Ernst Rank. The finite cell method for three-dimensional problems of solid mechanics. Computer methods in applied mechanics and engineering, 197(45):3768-3782, 2008.

[16] A. Ern and J.-L. Guermond. Theory and practice of finite elements, volume 159 of Applied Mathematical Sciences. Springer, 2004.

[17] Boyce E. Griffith, Richard D. Hornung, David M. McQueen, and Charles S. Peskin. An adaptive, formally second order accurate version of the immersed boundary method. Journal of Computational Physics, 223(1):10 - 49, 2007.

[18] Boyce E. Griffith and Charles S. Peskin. On the order of accuracy of the immersed boundary method: Higher order convergence rates for sufficiently smooth problems. Journal of Computational Physics, 208(1):75 - 105, 2005.

[19] E. Hachem, S. Feghali, R. Codina, and T. Coupez. Immersed stress method for fluid-structure interaction using anisotropic mesh adaptation. International Journal for Numerical Methods in Engineering, 94(9):805-825, 2013.

[20] Anita Hansbo and Peter Hansbo. An unfitted finite element method, based on nitsche's method, for elliptic interface problems. Computer Methods in Applied Mechanics and Engineering, 191(47):5537 - 5552, 2002.

[21] H. Huang and G. Scovazzi. A high-order, fully coupled, upwind, compact discontinuous galerkin method for modeling of viscous fingering in compressible porous media. Computer Methods in Applied Mechanics and Engineering, 263:169 - 187, 2013.

[22] Thomas J.R. Hughes, Arif Masud, and Jing Wan. A stabilized mixed discontinuous galerkin method for darcy flow. Computer Methods in Applied Mechanics and Engineering, 195(25):3347 - 3381, 2006. Discontinuous Galerkin Methods.

[23] Thomas J.R. Hughes, Guglielmo Scovazzi, Pavel B. Bochev, and Annalisa Buffa. A multiscale discontinuous galerkin method with the computational structure of a continuous galerkin method. Computer Methods in Applied Mechanics and Engineering, 195(19):2761 - 2787, 2006.

[24] M.P. Kirkpatrick, S.W. Armfield, and J.H. Kent. A representation of curved boundaries for the solution of the navier-stokes equations on a staggered three-dimensional cartesian grid. Journal of Computational Physics, 184(1):1 - 36, 2003.

[25] S. Lee, Y. Lee, and M. Wheeler. A locally conservative enriched galerkin approximation and efficient solver for elliptic and parabolic problems. SIAM Journal on Scientific Computing, 38(3):A1404-A1429, 2016.

[26] Randall J. LeVeque and Zhilin Li. Immersed interface methods for stokes flow with elastic boundaries or surface tension. SIAM Journal on Scientific Computing, 18(3):709-735, 1997.

[27] Mark N. Linnick and Hermann F. Fasel. A high-order immersed interface method for simulating unsteady incompressible flows on irregular domains. Journal of Computational Physics, 204(1):157 - 192, 2005.

[28] A. Main and G. Scovazzi. The shifted boundary method for embedded domain computations. part i: Poisson and stokes problems. Journal of Computational Physics, 2017.

[29] A. Main and G. Scovazzi. The shifted boundary method for embedded domain computations. part ii: Linear advection-diffusion and incompressible navier-stokes equations. Journal of Computational Physics, 2018.

[30] Arif Masud and Thomas J.R. Hughes. A stabilized mixed finite element method for darcy flow. Computer Methods in Applied Mechanics and Engineering, 191(39):4341 - 4370, 2002.

[31] Alireza Mazaheri and Hiroaki Nishikawa. Improved second-order hyperbolic residual-distribution scheme and its extension to third-order on arbitrary triangular grids. Journal of Computational Physics, 300:455 - 491, 2015.

[32] Alireza Mazaheri and Hiroaki Nishikawa. Efficient high-order discontinuous galerkin schemes with first-order hyperbolic advection-diffusion system approach. Journal of Computational Physics, 321:729 - 754, 2016

[33] Alireza Mazaheri, Mario Ricchiuto, and Hiroaki Nishikawa. A first-order hyperbolic system approach for dispersion. Journal of Computational Physics, 321:593 - 605, 2016

[34] Rajat Mittal and Gianluca Iaccarino. Immersed boundary methods. Annual Review of Fluid Mechanics, 37(1):239-261, 2005.

[35] Hiroaki Nishikawa. Robust and accurate viscous discretization via upwind scheme - i: Basic principle. Computers $\mathcal{E}$ Fluids, 49(1):62 - 86, 2011.

[36] Hiroaki Nishikawa. On hyperbolic method for diffusion with discontinuous coefficients. Journal of Computational Physics, 367:102 - 108, 2018

[37] J. A. Nitsche. Uber ein Variationsprinzip zur Losung Dirichlet-Problemen bei Verwendung von Teilraumen, die keinen Randbedingungen unteworfen sind. Abh. Math. Sem. Univ., Hamburg, 36:9-15, 1971.

[38] L. Nouveau, H. Beaugendre, C. Dobrzynski, R. Abgrall, and M. Ricchiuto. An adaptive, residual based, splitting approach for the penalized navier stokes equations. Computer Methods in Applied Mechanics and Engineering, 303:208 - 230, 2016.

[39] Jamshid Parvizian, Alexander Düster, and Ernst Rank. Finite cell method. Computational Mechanics, 41(1):121-133, 2007.

[40] Charles S Peskin. Flow patterns around heart valves: A numerical method. Journal of Computational Physics, $10(2): 252-271,1972$.

[41] Ramsharan Rangarajan and Adrián J Lew. Universal meshes: A method for triangulating planar curved domains immersed in nonconforming meshes. International Journal for Numerical Methods in Engineering, 98(4):236-264, 2014.

[42] W.H. Reed and T.R. Hill. Triangular mesh methods for the neutron transport equation. Technical report, Los Alamos Scientific Laboratory (NM), United States, 101973.

[43] B Schott, U Rasthofer, V Gravemeier, and WA Wall. A face-oriented stabilized nitsche-type extended variational multiscale method for incompressible two-phase flow. International Journal for Numerical Methods in Engineering, 104(7):721-748, 2015.

[44] B Schott and WA Wall. A new face-oriented stabilized xfem approach for $2 \mathrm{~d}$ and $3 \mathrm{~d}$ incompressible navier-stokes equations. Computer Methods in Applied Mechanics and Engineering, 276:233-265, 2014.

[45] G. Scovazzi, A. Gerstenberger, and S.S. Collis. A discontinuous galerkin method for gravity-driven viscous fingering instabilities in porous 
media. Journal of Computational Physics, 233:373 - 399, 2013.

[46] T. Song, A. Main, G. Scovazzi, and M. Ricchiuto. The shifted boundary method for hyperbolic systems: Embedded domain computations of linear waves and shallow water flows. Journal of Computational Physics, 369:45 - 79, 2018.

[47] Fotis Sotiropoulos and Xiaolei Yang. Immersed boundary methods for simulating fluid-structure interaction. Progress in Aerospace Sciences, $65: 1-21,2014$.

[48] H. S. Udaykumar, W. Shyy, and M. M. Rao. Elafint: A mixed eulerian-lagrangian method for fluid flows with complex and moving boundaries. International Journal for Numerical Methods in Fluids, 22(8):691-712, 1996.

[49] Guoyin Wang, Guglielmo Scovazzi, Léo Nouveau, Christopher E. Kees, Simone Rossi, Oriol Colomés, and Alex Main. Dual-scale galerkin methods for darcy flow. Journal of Computational Physics, 354:111 - 134, 2018

[50] Mary Fanett Wheeler. An elliptic collocation-finite element method with interior penalties. SIAM Journal on Numerical Analysis, 15(1):152161, 1978. 